\title{
Behavioral and Neurochemical Alterations in Mice Deficient in Anaplastic Lymphoma Kinase Suggest Therapeutic Potential for Psychiatric Indications
}

\author{
James G Bilsland', Alan Wheeldon ${ }^{2}$, Andrew Mead', Petr Znamenskiy', Sarah Almond ${ }^{2}$, Kerry A Waters ${ }^{2}$, \\ Matthew Thakur', Vahri Beaumont', Timothy P Bonnert', Robert Heavens', Paul Whiting', \\ George McAllister' and Ignacio Munoz-Sanjuan*,' \\ 'Department of Molecular and Cellular Neuroscience, Merck Sharp and Dohme, The Neuroscience Research Centre, Essex, UK; ${ }^{2}$ Department of \\ In vivo Neuroscience, Merck Sharp and Dohme, The Neuroscience Research Centre, Essex, UK
}

\begin{abstract}
The receptor tyrosine kinase product of the anaplastic lymphoma kinase (ALK) gene has been implicated in oncogenesis as a product of several chromosomal translocations, although its endogeneous role in the hematopoietic and neural systems has remained poorly understood. We describe that the generation of animals homozygous for a deletion of the ALK tyrosine kinase domain leads to alterations in adult brain function. Evaluation of adult ALK homozygotes (HOs) revealed an age-dependent increase in basal hippocampal progenitor proliferation and alterations in behavioral tests consistent with a role for this receptor in the adult brain. ALK HO animals displayed an increased struggle time in the tail suspension test and the Porsolt swim test and enhanced performance in a novel objectrecognition test. Neurochemical analysis demonstrates an increase in basal dopaminergic signalling selectively within the frontal cortex. Altogether, these results suggest that ALK functions in the adult brain to regulate the function of the frontal cortex and hippocampus and identifies ALK as a new target for psychiatric indications, such as schizophrenia and depression, with an underlying deregulated monoaminergic signalling.

Neuropsychopharmacology (2008) 33, 685-700; doi:I 0. I038/sj.npp. I 30I446; published online 9 May 2007
\end{abstract}

Keywords: ALK; dopamine; neurogenesis

\section{INTRODUCTION}

The regulation of neurogenesis has become the focus of considerable attention due to the extensive correlative evidence of the functional implications of newborn neuronal generation in the hippocampus, towards cognitive processes, and the potential involvement of increased proliferation of progenitors or neuronal generation in the mechanism of action of classical antidepressants (McEwen and Olie, 2005; Santarelli et al, 2003; Kempermann et al, 2004a; Cameron et al, 1998; Lie et al, 2004; Shors et al, 2001; Bruel-Jungerman et al, 2005; Snyder et al, 2005). However, the question of whether the observed increases in neuroprogenitor proliferation is an epiphenomenon of little functional relevance in the adult brain or a required event in the plethora of changes that ensue following pharmaco-

\footnotetext{
*Correspondence: Current address. Dr I Munoz-Sanjuan, Amgen Inc., II 20 Veterans Boulevard, South San Francisco, CA 94080, USA, Tel: + I 650244 2246, E-mails: imunoz@amgen.com or ignacio.munoz.sanjuan@gmail.com Received II September 2006; revised 9 February 2007; accepted 10 April 2007
}

logical treatment is still challenged. In addition, it has been well documented that neuroprogenitor cells located in the ventricular walls can respond to injury and neuronal degeneration in various models, including neuronal death induced by toxin administration (Felling and Levison, 2003; Magavi et al, 2000; Zhang et al, 2004; Nakatomi et al, 2002; Gould and Tanapat, 1997). This regulation, which happens certainly, albeit not exclusively, at the level of cell-cycle progression, highlights the maintenance of regenerative mechanisms in the adult central nervous system (CNS), which can be theoretically exploited towards clinical applications (Kempermann, 2002; Kempermann and Gage, 1999; Leuner et al, 2004; Gould and Tanapat, 1997).

Although several pathways have been implicated in the control of progenitor proliferation during development and in the adult (Kempermann et al, 2004a; Lie et al, 2004), little evidence has been obtained from genetic studies towards the identification of molecules with a regulatory role in this process, whether directly or indirectly. Some recent evidence suggests that there are mechanisms that restrict the proliferative capacity of either stem cells or rapidly expanding progenitors in the brain. For instance, loss of reverse signalling by the ephrin signalling pathway leads 
to a selective increase in ventricular zone progenitors (Holmberg et al, 2005) and dopamine modulation has been postulated to regulate baseline proliferation in an inhibitory manner (Kippin et al, 2005), although various studies suggest that the role of dopamine modulation in the regulation of neurogenesis might be more complex (Ohtani et al, 2003; Hoglinger et al, 2004).

Interestingly, most studies aimed at the modulation of classical neurotransmitter systems, or neuroendocrine control, have identified a threshold that correlates with functional efficacy of such treatments in neurophysiological modulation. For instance, the observed expansion of hippocampal progenitors on chronic antidepressant treatment or neurotransmitter modulation is typically confined within a $30-60 \%$ increase in cells immunoreactive (IR) for S-phase markers, such as bromo-deoxyuridine (BrdU) or the proliferating cell nuclear antigen (PCNA) in either the subgranular zone (SGZ) or subventricular zone (SVZ) (Santarelli et al, 2003; Mirescu et al, 2004; Hoglinger et al, 2004; Monje et al, 2003; Kippin et al, 2005). Similarly, in studies with chronic corticosterone administration or in stress paradigms (Mirescu et al, 2004; Bilsland et al, 2006), the negative effects on progenitor proliferation are within a similar range, potentially highlighting a physiological window towards the regulation of progenitor cell-cycle regulation.

To identify novel targets and mechanisms that might be of relevance to psychiatric disease, we analyzed a collection of mouse knockout (KO) animals for changes in baseline hippocampal proliferation, using a fluorescent-activated cell sorting (FACS)-based method to detect changes in BrdU incorporation in progenitors (Bilsland et al, 2006). In this study, we present evidence for the identification of a gene via such an approach, the anaplastic lymphoma kinase (ALK), whose loss of function leads to an age-dependent change in hippocampal progenitor proliferation. ALK is a member of the insulin-receptor superfamily and several putative ligands have been described, including the secreted factors midkine and pleiotrophin, factors with a known effect in neuronal maturation, neurite outgrowth, and the regulation of synaptic plasticity (Amet et al, 2001; Nakamura et al, 1998; Zhou et al, 1997; Kinnunen et al, 1996). We demonstrate that the ALK KO animals display an antidepressant profile in behavioral paradigms, increased basal dopaminergic tone in the prefrontal cortex, and enhanced performance in a hippocampal-dependent task, without any alterations in locomotor activity. This occurs in the absence of any apparent developmental or anatomical deficits in the brain. Altogether, these results highlight the involvement of ALK function in the regulation of behavior in the adult mouse and suggests that antagonism of ALK activity in the adult might constitute a novel pharmacological strategy towards the treatment of cognitive and/or mood disorders.

\section{METHODS}

\section{ALK KO Generation and Genotyping}

ALK homozygote (HO) mice were obtained from Deltagen (San Carlos, CA). A 6.93-kb IRES- $\beta$-galactosidase $(\beta$-gal) reporter and neomycin-resistance cassette (IRES- $\beta$-gal-neo) was subcloned into a 9.2-kb fragment isolated from a mouse genomic phage library, such that $35 \mathrm{bp}$ coding for the protein were replaced by IRES- $\beta$-gal-neo. The IRES- $\beta$-galneo cassette was flanked by $4.0 \mathrm{~kb}$ of mouse genomic DNA at its $5^{\prime}$ aspect and $5.2 \mathrm{~kb}$ of mouse genomic DNA at its $3^{\prime}$ aspect. The targeting vector was linearized and electroporated into 129/OlaHsd mouse embryonic stem (ES) cells. ES cells were selected for G418 resistance and colonies carrying the homologously integrated neo DNA were identified by PCR amplification using a $5^{\prime}$ neo-specific primer (5'-GGGATCTTGGCCATGGTAAGCTGAT- $\left.3^{\prime}\right)$ paired with a primer located outside the targeting homology arms on the $5^{\prime}$ side (GS1: 5'-CCAAGAGAGGACTGAAGAAGTG GG-3'). The homologous recombination event was confirmed on the $3^{\prime}$ side using a $3^{\prime}$ neo-specific primer $\left(5^{\prime}-\mathrm{ACG}\right.$ TACTCGGATGGAAGCCGGTCTT- $3^{\prime}$ ) paired with a primer located outside the targeting homology arm on the $3^{\prime}$ side. (GS2: 5'-GTGAATCTTCCTCATGTTCCAGAGCC-3'). Colonies that gave rise to the correct size PCR product were confirmed by Southern blot analysis using a probe adjacent to the $3^{\prime}$ region of homology. The presence of a single neo cassette was confirmed by Southern blot analysis using a neo gene fragment as a probe. Male chimeric mice were generated by injection of the targeted ES cells into C57Bl/ $6 \mathrm{~J}$ blastocysts. Chimeric mice were bred with $\mathrm{C} 57 \mathrm{Bl} / 6 \mathrm{~J}$ mice to produce F1 heterozygotes (HEs). Germline transmission was confirmed by PCR analysis. Initial germline HEs were also tested for the homologous recombination event using the primers described above (located outside of the targeting construct). Following confirmation of the targeting event in animals, subsequent genotyping tracked transmission of the targeting construct. F1 heterozygous males and females were mated to produce F2 wild-type (WT), heterozygous, and homozygous null mutant animals. Mice were backcrossed with C57BL/6J mice and all phenotypic analyses were performed in a hybrid C57Bl/6J/129 background (75/25\%). Mice were maintained in a temperature-controlled $\left(23^{\circ} \mathrm{C}\right)$ barrier facility with a 12-h light-dark cycle and had ad libitum access to water and regular rodent chow.

\section{FACS-Based BrdU Quantification. Analysis of Halogenated Thymidine Analogs}

Animals were killed by stunning and decapitating. One hemisphere was immersion fixed in $10 \%$ formal saline for $72 \mathrm{~h}$ for immunohistochemistry and the remaining half was dissected to isolate the hippocampal lobe. FACS analysis was performed as described in Bilsland et al (2006). BrdU was dosed via the intraperitoneally (i.p.) route at $100 \mathrm{mg} / \mathrm{kg}$. Thymidine analogues were administered at the concentration equimolar to $50 \mathrm{mg} / \mathrm{kg}$ BrdU: IdU at $57.5 \mathrm{mg} / \mathrm{kg}$ and CldU at $42.6 \mathrm{mg} / \mathrm{kg}$. All procedures were carried out according to Home Office guidelines. For the CldU and IdU studies, two doses of IdU were given followed by two doses of CldU on subsequent days. The animals were killed 2 days after the last dose. Fluorescence immunohistochemistry was used for the detection of both analogues in same sections.

\section{QPCR, Immunohistochemistry and X-gal Staining}

Quantification of transcript analysis of ALK expression was detected with Taqman probes designed to amplify the 
following regions: N-terminal probe, nucleotides 591-655; C-terminal probe 2, nucleotides 4502-4562, and C-terminal probe 3, nucleotides 4319-4388. Data are presented as the result of a comparison of tissues derived from $n=6$ animals for each HOs, relative to the expression in the WT controls for each tissue. Each sample is normalized by the expression of $\alpha$-tubulin. Transcript levels for midkine, pleiotrophin, the receptor-protein phosphatase PTPR-Z1, the dopamine receptors D2 and D1, the serotonin receptor 5-HT1a, and the signal transducer DARPP-32 were determined as in Bonnert et al (2006) using standard Taqman procedures and are plotted as relative expression values in the ALK HO animals, normalized to WT levels in both the prefrontal cortex or hippocampus.

For BrdU immunocytochemistry studies, fixed hemispheres were embedded in paraffin and sectioned to $6 \mu \mathrm{m}$ using a rotary microtome (Leitz, Nussloch, Germany) and mounted on Fisher Superfrost Plus slides (BDH, Poole, UK). Sections were dewaxed in xylene, incubated in $0.3 \%$ hydrogen peroxide in PBS to quench endogenous peroxidase, washed for $3 \mathrm{~min}$ in PBS, and then microwaved for up to $10 \mathrm{~min}$ in $10 \mathrm{mM}$ citrate buffer, $\mathrm{pH} 6.0$, to retrieve antigenicity (Shi et al, 1991). Sections were blocked in 5\% normal serum, matched to the species the secondary antibody was raised in, in $0.01 \mathrm{M}$ PBS containing $0.03 \%$ Triton X-100, before overnight incubation at $4{ }^{\circ} \mathrm{C}$ in affinitypurified antiserum made up in species-matched normal serum. Immunoreactivity was detected using $30 \mathrm{~min}$ incubation with biotinylated secondary antibody (Vector Laboratories, Peterborough, UK) and $30 \mathrm{~min}$ ABC (Vector) made up in Optimax buffer (Biogenex) and subsequently visualized using diaminobenzidine/hydrogen peroxide (Menarini A Diagnotics, Wokingham, UK) for $10 \mathrm{~min}$. All immunohistochemical detection steps (from secondary antibody stage onwards) were performed on a Biogenex Optimax robotic immunostainer (model 1.5v). Sections were counterstained with hematoxylin. Sections were imaged using a Leica Leitz DMRD light microscope, in some cases using diffusion interference contrast (Leica Microsystems Ltd, Milton Keynes, UK). An experimenter, blinded to the identity of the sections, counted labelled cells at $\times 100$ and $\times 400$ under a light microscope (Leica Microsystems Ltd). For cell counting in the dentate gyrus (DG), every 10th section throughout the whole hippocampus was processed for immunohistochemistry. A cell is counted as being in the SGZ of the DG if it is touching or in the SGZ, which is defined as the region of the granular zone within three cell depths from the hilus. The total number of counted cells was then multiplied by the sampling fraction to give a count for the whole DG. Cell counts were compared using an unpaired Student's $t$-test with a $p$-value of 0.05 .

For the halogenated thymidine analogue study, we first verified that individual antibodies did not cross-react by monitoring immunoreactivity in singly dosed animals, with HRP detection. The sections were antigen retrieved by boiling in citrate buffer ( $\mathrm{pH} \mathrm{6}$ ) for $10 \mathrm{~min}$, which unmasked the tissue and denatured the DNA. The sections were then blocked for endogenous mouse IgG with a vector mouseon-mouse kit and for nonspecific antibody binding with $0.1 \% \mathrm{BSA}$ for $30 \mathrm{~min}$. The primary antibodies diluted in $0.1 \%$ BSA were then incubated for $1 \mathrm{~h}$. Biotin-streptavidin amplification was applied to increase the signal-to-noise ratio for CldU. For immunofluorescent staining, AlexaFluor 488-conjugated anti-mouse antibody (rat adsorbed) and biotinylated anti-rat antibody (mouse absorbed) with AlexaFluor 568-conjugated streptavidin were used. The sections were counter stained with TOTO3 (1:1000 for 30 s) and coverslipped with Vectashield HardSet mounting medium with DAPI. All secondary reagents and conjugates were used at 1:200 dilutions and incubated for $30 \mathrm{~min}$. All washes and dilutions were done in Biogenex Super Sensitive wash buffer. HRP-stained sections were used for quantification and examined using a light microscope. All counts were done at $\times 400$ magnification to distinguish cells within clusters. The number of IR cells was estimated by an experimenter blinded to the identity of the sections. Every 40th section spanning the whole of the hippocampus was examined.

Antibodies used were as follows: rat anti-BrdU (Serotec mca2060, $1: 200$ dilution); Abcam mouse anti-PCNA (ab29$100 ; 1: 1600,5$ min retrieval); Becton-Dickinson mouse antiBrdU, 1 : 400 for IdU; Abcam rat anti-BrdU, 1:800 for CldU; Serotec, Santa Cruz goat anti-doublecortin (C-18; 1:300, 10 min retrieval); Chemicon rabbit anti-calretinin $(1: 5000$, $10 \mathrm{~min}$ retrieval); Abcam mouse anti-calbindin (ab9481500; $1: 400,5$ min retrieval); Sigma mouse anti-parvalbumin (p3088; $1: 2500,10$ min retrieval); Invitrogen rabbit anti- $\beta$ gal ( $1: 400$ with $10 \mathrm{~min}$ retrieval).

For X-gal double staining with NeuN and GFAP antibodies, X-gal-stained sections (conventional staining as whole mounts) were frozen. Sections were brought to room temperature, postfixed in acetone for $5 \mathrm{~min}$ and quenched for endogenous peroxidase activity in $0.3 \% \mathrm{H}_{2} \mathrm{O}_{2}$ for $30 \mathrm{~min}$. They were then blocked with $5 \%$ normal goat serum for $1 \mathrm{~h}$ and incubated with rabbit anti-GFAP antibody $1: 3000$ (ABR OPA1-06100) or anti-NeuN (Chemicon mab377, 1:10000) at $4{ }^{\circ} \mathrm{C}$ overnight. Secondary goat anti-rabbit antibody (Vector Labs) was applied for $30 \mathrm{~min}$. From this step the protocol is identical to BrdU staining. The sections were counterstained with Neutral Red for 2 min.

\section{SVZ Dissociation, Culturing and Proliferation Assays}

Animals were killed by stunning and cervical dislocation. Tail snips were also taken for reconfirmation of genotype. Brains were removed and placed on wet paper towel (soaked in HBSS). Cuts were made 1-2 mm behind the rostral tip of the brain using a scalpel and another cut made 1-2 mm caudally. The ensuing slice was placed with the rostral surface facing up and a cut was made using forceps through the corpus callosum. Cortex was peeled back, exposing the striatal surface of ventricle, and a thin strip of tissue removed into HBSS; SVZ is contained within this tissue. Each animal was killed and dissected independently to minimize postmortem interval before tissue collection. SVZ samples from each animal were placed independently into 24-well plate wells and processed independently thereafter. After all brains were dissected, tissue from each animal was placed into Eppendorf tubes containing $0.5 \mathrm{ml}$ HBSS-glucose solution with trypsin, hyaluronidase, kynurenic acid, and DNase I for $30 \mathrm{~min}$. After $15 \mathrm{~min}$, samples were triturated 10 times with a 1-ml Gilson and triturated 
once more at $30 \mathrm{~min}$. EBSS-HEPES-BSA $(0.5 \mathrm{ml})$ solution was added and samples filtered through $70 \mu \mathrm{m}$ cell strainers into another set of Eppendorf tubes, ensuring no crosscontamination of samples derived from individual animals. Samples were spun $5 \mathrm{~min}$ at 1250 r.p.m. in centrifuge, then supernatant aspirated and samples resuspended in $1 \mathrm{ml}$ HBSS-sucrose solution. Samples were spun at 2500 r.p.m. to pellet, then supernatant aspirated and samples resuspended in $200 \mu \mathrm{l}$ DMEM:F12 1:1 supplemented with B27, PSF, epidermal growth factor (EGF), and fibroblast growth factor (FGF)2 $20 \mathrm{ng} / \mathrm{ml}$ (growth medium). Resuspended samples were layered onto $1.2 \mathrm{ml}$ HBSS-BSA-HEPES solution and spun at 1250 r.p.m. for $7 \mathrm{~min}$. This supernatant was aspirated and cells were resuspended in $1 \mathrm{ml}$ growth medium and plated into 24-well plates for neurosphere expansion. Spheres were fed at $4 \mathrm{div}$ by the addition of $500 \mu \mathrm{l}$ growth medium to the cultures. Number of primary neurospheres was quantitated visually after 8 days in culture. After quantification, spheres were fed again by $50 \%$ medium change.

For secondary sphere analysis, primary spheres from each animal were dissociated individually and collected in $1.5 \mathrm{ml}$ Eppendorff tubes. Tubes were spun at 1250 r.p.m. for 2 min to pellet spheres. Supernatant was removed and spheres resuspended in d-PBS ( $1 \mathrm{ml} /$ sample) and centrifuged as before. Accutase $(200 \mu \mathrm{l})$ was added per sample and spheres were resuspended by triturating five times with a 200- $\mu$ l Gilson tip (changed between every sample). Samples were incubated at $37^{\circ} \mathrm{C} / 5 \% \mathrm{CO}_{2}$ for $15 \mathrm{~min}$, then samples triturated 10 times as before. Samples were incubated up to $30 \mathrm{~min}$ and then triturated five times. D-PBS $(1 \mathrm{ml})$ was added to each sample and the samples were centrifuged at 1250 r.p.m. for $5 \mathrm{~min}$. Samples were resuspended in $1 \mathrm{ml}$ growth medium and $50 \mu \mathrm{l}$ was transferred to each window of a hemocytometer. Phase-bright cells were counted on inverted microscope; four grids were counted for each sample. Cells were plated at clonal densities in 96-well plates for secondary neurosphere quantifications ( 1 cell/ $\mu \mathrm{l})$. After 8 days in culture, the number of individual spheres was quantified by a blinded experimenter.

For the proliferation studies with ALK and WT neurospheres in different growth factor backgrounds, cells were dissociated as above at various passages and plated clonally (density of 5 cells/ $\mu \mathrm{l}$ in 96-well plates), with either no growth factors (DMEM + B27 + Penn/Strep), FGF2 (20 ng/ml), EGF $(20 \mathrm{ng} / \mathrm{ml})$, or medium containing both EGF/FGF2 $(20 \mathrm{ng} / \mathrm{ml}$ each). Cells were incubated for 3 days and then the cell number was estimated based on overall adenosine triphosphate (ATP) content assayed using the Promega CellTiter$\mathrm{Glo}^{\mathrm{TM}}$ luminescent cell viability assay (G7572). Cells were equilibrated to room temperature before the addition of reagent and incubated for $5 \mathrm{~min}$ before counting in Topcount. Typically, $n=6$ or 12 wells/condition were counted and averaged after a 48 - or 72 -h incubation period.

\section{Behavioral Studies}

All animal procedures were performed in accordance with the UK Animals (Scientific Procedures) Act, 1986.

Mice were habituated for 1 week and weighed-matched at approximately $25 \mathrm{~g}$. All animals used in the behavioral studies were between 2-8 months of age. They were individually housed, with food and water available ad libitum. Animals were maintained in a temperature- and humidity-controlled environment $\left(20-23^{\circ} \mathrm{C} ; 45-60 \%\right.$ humidity) with a 12-h light-dark cycle. We detected no significant effects of sex in any of the behavioral studies performed and therefore data from males and females were pooled for the final analysis.

\section{Locomotor Activity Test}

For the locomotor, tail suspension test (TST), and Porsolt swim test (PST), $n=11$ ALK HOs (nine female and two males) and $n=8$ WT (four males and four females) mice of 6-8 months were used (see below). Mice were individually placed in a white Perspex arena $(30 \times 27 \times 30 \mathrm{~cm})$. The floor was divided into $12(4 \times 3)$ boxes, each $(8.5 \times 7.5 \mathrm{~cm})$ defined by a black line. The mice were observed for $10 \mathrm{~min}$ under normal lighting conditions and the number line crossings recorded.

\section{TST}

Mice were individually sellotaped by the tail to a wooden bar held horizontally $45 \mathrm{~cm}$ from the bench. Mice would initially struggle and then show increased spates of immobility. A trained observer measured the duration of immobility over a 5-min period. Statistical analysis was carried out using the immobility scores. Student's $t$-test was used to compare the duration of immobility by $\mathrm{HO}(n=11)$ and WT $(n=8)$ mice. Significance was attributed when $p<0.05$.

\section{PST}

Swim tests were conducted in a glass cylinder of water $\left(29-30^{\circ} \mathrm{C}\right)$ the level of the water was such that it does not allow for the hind limbs or tail to touch the bottom. Mice were placed in the Porsolt apparatus for $5 \mathrm{~min}$. Escape, swim, or immobility behavior was recorded by video camera. A trained observer then subsequently viewed the videotape and recorded the duration of each behavior. The tape was scored blind. Statistical analysis was carried out using the immobility scores. ALK KO and WT mice were compared using Student's $t$-test. Significance was attributed when $p<0.05$.

\section{Black/White Anxiety Test}

Animals, 2-3-month-old, were placed in an open-top test box $(45 \times 27 \times 27 \mathrm{~cm})$ that was divided by a partition. The smaller chamber $(15 \times 27 \times 27 \mathrm{~cm})$ was colored black and the larger chamber $(30 \times 27 \times 27 \mathrm{~cm})$ white. Between the two was a partition with a $7.5 \times 7.5 \mathrm{~cm}$ central opening that allowed mice to move between compartments. The white compartment was illuminated by lamps, giving approximately 450 lux on the light side. The dark side was illuminated by red light (light intensity 7 lux) to facilitate tracking with an infrared camera. Mice were placed individually in the white area, facing away from the black section and the time spent in the white section observed by remote video recording for $10 \mathrm{~min}$. Time spent in each compartment was recorded using HVS Image VP200 tracker boxes (sampling rate $0.1 \mathrm{~s}$ ) and HVS Field software. ALK KO and WT mice were compared using Student's $t$-test. Significance was attributed when $p<0.05$. 
Novel Object Recognition (NOR)/Novel Object Location (NOL) Test

Male and female ALK HO $(n=10)$ and WT $(n=8)$ mice of 6-8 months of age were used. They were group-housed with food and water available at all times. They were maintained in a temperature- and humidity-controlled environment $\left(20-23^{\circ} \mathrm{C} ; 45-60 \%\right.$ humidity) in a 12 -h light-dark cycle with lights on at 0700 hours. One $\mathrm{HO}$ mouse was excluded from the study due to very low levels of exploration in the sample trials. The methods used were similar to those described by Mandillo et al (2003). The protocol consisted of seven test sessions, each separated by a 2-min delay during which mice were returned to their home cage. During each test session, mice were placed in the test box for $6 \mathrm{~min}$.

Session 1-habituation (no objects).

Session 2-sample 1, five different objects placed in the test box, time spent exploring objects scored.

Session 3-sample 2.

Session 4-sample 3.

Session 5-(NOL) two objects switched locations, time spent exploring objects in novel locations compared with time spent exploring objects that had not been moved.

Session 6-same object arrangement as session 5, exploration on all objects scored.

Session $7-($ NOR) one object replaced by a new object, time spent exploring the novel object compared with time spent exploring the familiar objects.

All testing were carried out under red light. The test box measured approximately $50 \times 30 \mathrm{~cm}$ with the corners rounded off. Most of the inner box was gray, but a section was lined with a distinct black/white checkerboard design to facilitate the spatial determination of the objects within the test box. The objects used differed in their size, shape, and material such that they were all distinct. Objects were cleaned with ethanol between trials to avoid odor cues. Exploration was scored as time spent sniffing an object. Sitting on or climbing over an object was not scored. The amount of exploration per object was calculated during each session to offset differing numbers of novel and familiar objects in each trial. Exploration in session 4 (preNOL) was considered to be baseline exploration for the NOL session, such that exploration was scored on the objects that were to remain unchanged in session 5 (familiar) and also for the two objects that were to be switched in session 5 (novel). Similarly, in session 6 (pre-NOR) the baseline scores for the novel and familiar objects were calculated before the NOR session. In this way, the pre-NOL and-NOR data could be compared with the scores in the NOL and NOR sessions, respectively, enabling an actual level of exploration to be calculated that was not influenced by the object itself or its position within the box. The net time spent exploring the novel object (d1 score) was calculated. The d1 scores were analyzed using a repeated measures ANOVA, followed by Tukey's post hoc test in Statistica. Significance was attributed when $p<0.05$.

\section{HPLC Analysis of Dopamine, Serotonin, and Metabolites}

Prefrontal cortex $(n=6 \mathrm{WT} ; n=4 \mathrm{KO})$, hippocampal $(n=17 \mathrm{WT}$ and $n=7 \mathrm{KO})$, and striatal $(n=8 \mathrm{WT}$ and $n=7 \mathrm{KO})$ samples obtained from several cohorts (2-5month-old) were dissected from two separate cohorts of adult animals and stored at $-80^{\circ} \mathrm{C}$ immediately. Data were pooled for the analysis. Samples from medial prefrontal cortex, striatum, and hippocampus were analyzed for serotonin and the metabolite 5HIAA by high-performance liquid chromatography (HPLC) voltametry, essentially as described in Hutson et al, 1991. Samples were homogenized in 10 volumes of homogenizing buffer $(0.4 \mathrm{M}$ perchloric acid, $0.1 \%$ cysteine, $0.1 \%$ sodium metabisulphite, $0.01 \%$ NaEDTA) and centrifuged at $10000 \mathrm{~g}$ for $10 \mathrm{~min}$, supernatants were collected and analyzed by HPLC with electrochemical detection (Hutson et al, 1991). The HPLC system comprised a Jasco PU-980 pump, a Waters 717 plus autosampler, a Waters Spherisorb ODS column $(2 \times 100 \mathrm{~mm})$, and an Antec Intro electrochemical detector with the glassy carbon working electrode set at $+0.75 \mathrm{~V}$ relative to a silver/silver chloride reference electrode. The mobile phase consisted of $0.07 \mathrm{M} \mathrm{KH}_{2} \mathrm{PO}_{4}, 0.0035 \%$ Na2EDTA, $0.03 \%$ octyl sodium sulfate, and $10 \%$ methanol; $\mathrm{pH} 2.75$ at a flow rate of $0.3 \mathrm{ml} / \mathrm{min}$. Samples were analyzed using the Chromperfect software package (Justice Innovations) and integrated peak areas were related to standards containing $100 \mathrm{ng} / \mathrm{ml}$ each of dihydroxyphenylacetic acid (DOPAC), dopamine, homovanillic acid (HVA), 5-HIAA, and 5-HT diluted in tissue-homogenizing buffer. Data were calculated as $\mathrm{ng} / \mathrm{g}$ wet weight tissue and expressed as mean \pm SEM, values were compared with WT littermates. A one-way ANOVA was carried out with Dunnett's post hoc test to determine statistical significance using GraphPad Prism (version 3.03, GraphPad software Ltd).

\section{RESULTS}

\section{Generation of ALK Gene KO and Overall Phenotypic Characterization}

The ALK gene was targeted by conventional insertional mutagenesis of the $\beta$-gal gene, introducing a 35-bp deletion in exon 22 of the ALK locus in C57Bl/6J mice (Figure 1), leading to premature transcript termination and deletion of the transmembrane and kinase domains. Animals were genotyped (Figure 1) and ALK HO animals were analyzed for overall histopathology and body-fluid samples. ALK HOs are fertile and display a normal appearance. Detailed blood and urine sample analysis revealed no overt abnormalities and rough histochemical analysis revealed no obvious abnormalities in any tissue analyzed (data not shown), including the brain. In addition, no effects were observed in hematopoietic populations (data not shown), as might have been predicted, based on the expression and role of ALK in a variety of lymphomas (Coluccia et al, 2005; Duyster et al, 2001; Morris et al, 1997). To assess the effects of the KO strategy on ALK expression, we monitored ALK mRNA relative levels in various brain regions of $\mathrm{HO}$ and WT littermate animals, by quantitative reverse transcription-polymerase chain reaction (qPCR). To assess whether the insertional mutagenesis strategy lead to a complete ablation of ALK expression, we monitored ALK transcripts with probes designed to amplify various regions of this gene. The N-terminal probe amplifies nucleotides 591-655 and is upstream from the knocked-in $\beta$-gal gene. Two 

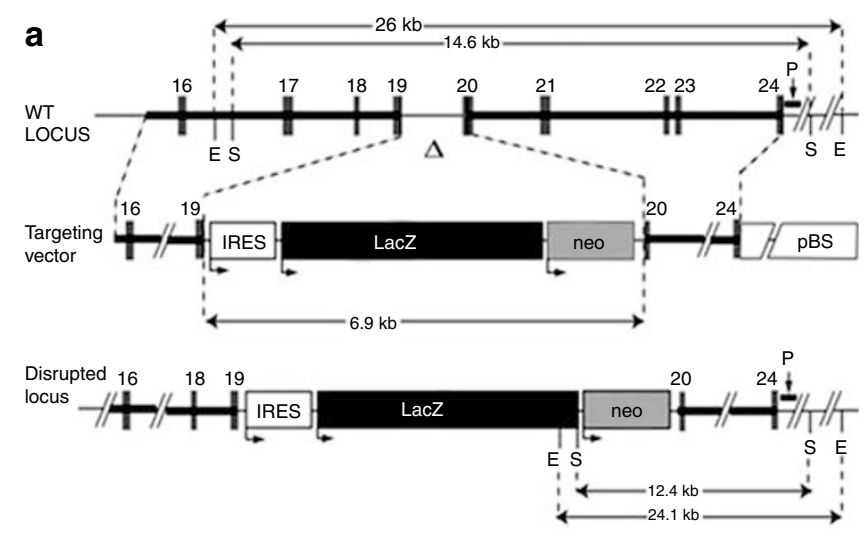

b HO WT WT HO HE WT WT HO

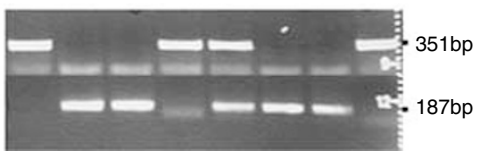

Figure I Generation of ALK KO mice. (a) Gene deletion strategy. (b) Genotyping of ALK receptor KO mice. Typical gel electrophoresis example showing homozygous mice with the $35 \mathrm{I}$ bp targeted product only, WT mice with the $187 \mathrm{bp}$ endogenous product only, and heterozygous with both products. $\mathrm{HO}$, homozygous; $\mathrm{HE}$, heterozygous; WT, wild-type mice.

C-terminal probes, termed C-terminal probes 2 and 3, amplify nucleotides $4502-4562$ and $4319-4388$, respectively. These probes detect transcript expression downstream of the insertion site and therefore there should not be any expression in the HO animals. Interestingly, the relative expression of these probes in the WT brain varies within any one tissue, the $\mathrm{N}$-terminal probe being the most highly expressed and the $\mathrm{C}$-terminal probe 3 the least abundant, in all brain regions examined (Figure 2a). This relative distribution argues for the expression of multiple $\mathrm{C}$-terminal splice variants and is evident in the relative expression of the C-terminal probe 2, which is lowest in the frontal cortex. The relative expression of ALK transcripts (as judged by the N-terminal probe) appears highest in the frontal cortex and cerebellum, roughly 9.3- and 2.6-fold higher than in the hippocampus, respectively (Figure $2 \mathrm{a}$ ). This difference in expression may be due to alternative splicing or transcript stability, although we cannot rule out an effect of the internal normalization to $\alpha$-tubulin, which we assume is constant across brain regions. This relative degree of ALK expression determined by qPCR across various brain regions is consistent with internal microarray data obtained from a mouse-body atlas project (see Bonnert et al, 2006). In this analysis, ALK transcript expression in the brain is highest in the frontal cortex and hypothalamus and the cerebellum and hippocampus show similar expression levels (data not shown).

Levels of the two $3^{\prime}$ probes were relatively lower in the frontal cortex suggesting potential alternatively spliced transcripts in this cortical area. Our analysis confirmed loss of expression of ALK C-terminal transcripts downstream of the insertion site, as expected from the targeting strategy, in all brain-tissues analyzed (Figure 2a). However, we could detect low level of expression of these probes and therefore we feel that this HO line probably represents a hypomorphic allele of ALK, at least at the molecular level. Interestingly, a Relative Expression of Each ALK Probe in Adult Mouse Brain Tissues

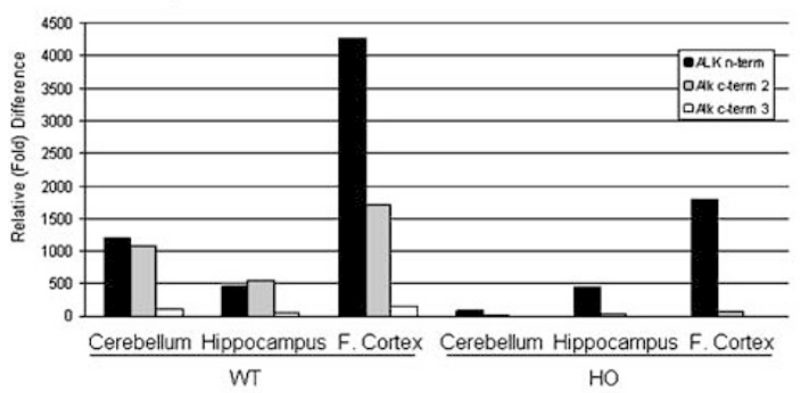

b Relative Transcript Expression - $\mathrm{HO}$ levels norrmalized to WT mice

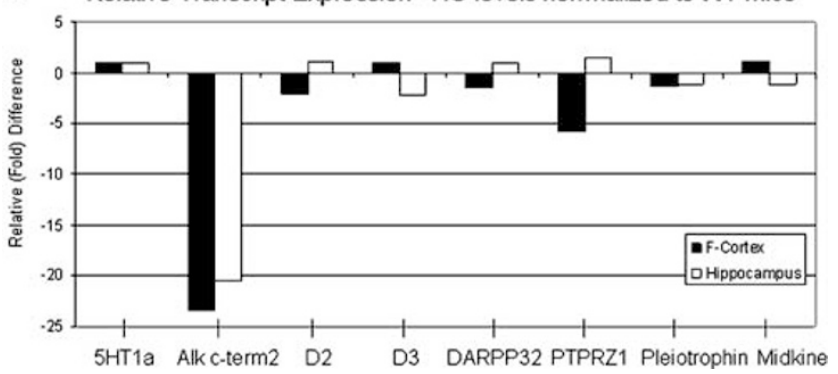

Figure 2 (a) Quantitative analysis of relative expression levels of ALK transcripts in cerebellum, hippocampus, and frontal cortex in HOs vs WT littermates ( $n=6$ animals/genotype). Data are normalized to $\alpha$-tubulin and expressed as relative fold-change. Notice that the relative expression of ALK transcripts (as judged by the N-terminal PCR probe) is higher in the frontal cortex and the cerebellum than in the hippocampus (9.3- and 2.6fold higher compared with hippocampal levels of this probe). Quantification of ALK products by qPCR in the $\mathrm{HO}$ animals shows lack of expression of mRNA transcripts encoding the $3^{\prime}$ end of ALK (c-2 and c-3 probes), as expected from the gene disruption strategy. Notice varying amounts of residual $5^{\prime}$ transcript across these brain regions. (b) Relative transcript expression of various genes implicated in ALK signalling or neurochemical pathways affected in the $\mathrm{HO}$ animals. Data is plotted as relative expression changes in $\mathrm{HO}$ vs WT animals in the frontal cortex and hippocampus. With the exception of ALK transcripts, we detected no statistically significant changes in any of the additional genes monitored.

when we monitored the expression of a probe designed to detect exon 2 (N-terminal probe), unaltered expression remains in the hippocampus, whereas the expression is absent in the cerebellum and reduced by half in the frontal cortex. Based on this analysis, we cannot conclude that a complete loss of ALK protein has been achieved and the possibility remains that truncated N-terminal fragments of ALK might be produced. To assess the specificity of the mutagenesis strategy and to evaluate whether there are any molecular alterations in the expression of additional components of the ALK signalling pathways (such as the ligands midkine and pleiotrophin or the receptor RPTP-Z) or various neurochemical pathways operative in these brain regions (such as serotonin and dopamine receptors and signal transducers), we performed qPCR for a subset of such genes and observed no statistically significant effects in gene expression in the frontal cortex or hippocampus in the $\mathrm{HO}$ animals (Figure 2b).

Attempts to use commercial antibodies to ALK or activated ALK protein did not yield specific and reproducible results. Therefore, we are unable to provide information regarding the levels of ALK protein made in this line. Instead, to further map the expression of ALK in the adult brain, we monitored endogeneous cellular expression of 
ALK by measuring the expression of the knocked-in $\beta$-gal transgene in ALK HEs (Figure 3). Similar expression patterns were observed in $\mathrm{HO}$ and $\mathrm{HE}$ animals, suggesting that loss of ALK function does not lead to cell death of expressing cells, at least in a noticeable manner. Expression was monitored in HE animals and was consistent with previous reports on ALK transcript expression during development and in the adult (Iwahara et al, 1997; Vernersson et al, 2006). In these reports, ALK expression was robust throughout the cortex, olfactory bulb, mesencephalic structures, and several thalamic nuclei. In our hands, $\beta$-gal expression was highest in the olfactory bulb and throughout the forebrain, including scattered cells in the frontal cortex, DG, CA1-CA3 layers of the hippocampus (Figure 3), the caudate putamen, amygdala (Figure 2 and data not shown), and throughout the thalamus and basal ganglia. We detected lower level of expression in the cerebellum and brainstem regions, in contrast with the data obtained by qPCR in the WT animals (Figure 2a) and the microarray data, which shows robust expression in these regions. We believe that this discrepancy might be due to the insertional mutagenesis employed to generate the $\mathrm{HO}$ animals. In the HO animals, the expression level of the N-terminal probe is lowest in the cerebellum (Figure 2a). As the $\beta$-gal expression arises from the $5^{\prime}$-end of the ALK gene, it may be expected to better resemble the $\mathrm{N}$-terminal ALK probe in terms of its expression. If this is indeed the case, expression of $\beta$-gal would show that there is low expression in the cerebellum compared with the hippocampus and the cortex, which is what we see in these animals. Therefore, the insertion strategy might have altered the relative expression of the different ALK transcripts. Alternatively, the limited pattern of expression of $\beta$-gal in a subset of pyramidal neurons in the CA3-CA1 layers of the hippocampus might partially reconcile the discrepancy with the $\mathrm{qPCR}$ data reported here.

Overall, $\beta$-gal expression was confined to mature neurons, as attested by double staining between X-gal staining and NeuN or GFAP antibodies. Similarly, in the hippocampus, scattered pyramidal cells in CA1-CA3 were immunorective for $\beta$-gal. A small subset of cells located in the SGZ of the DG were $\beta$-gal-positive, although these cells were not dividing as judged by BrdU-incorporation experiments, suggesting that hippocampal neuronal progenitor cells do not express ALK transcripts. The site of strongest expression, as judged by $\beta$-gal expression, is the olfactory bulb, including a subset of the mitral cells within the inner plexiform layer and presumed tufted cells in the external plexiform layer (Figure 3). Throughout the cortex, scattered neurons expressed $\beta$-gal, with stronger expression in frontal, entorhinal, and perirhinal cortical areas. Thalamic and hypothalamic nuclei were also sites of strong expression (data not shown), as reported previously (Iwahara et al, 1997; Vernersson et al, 2006).

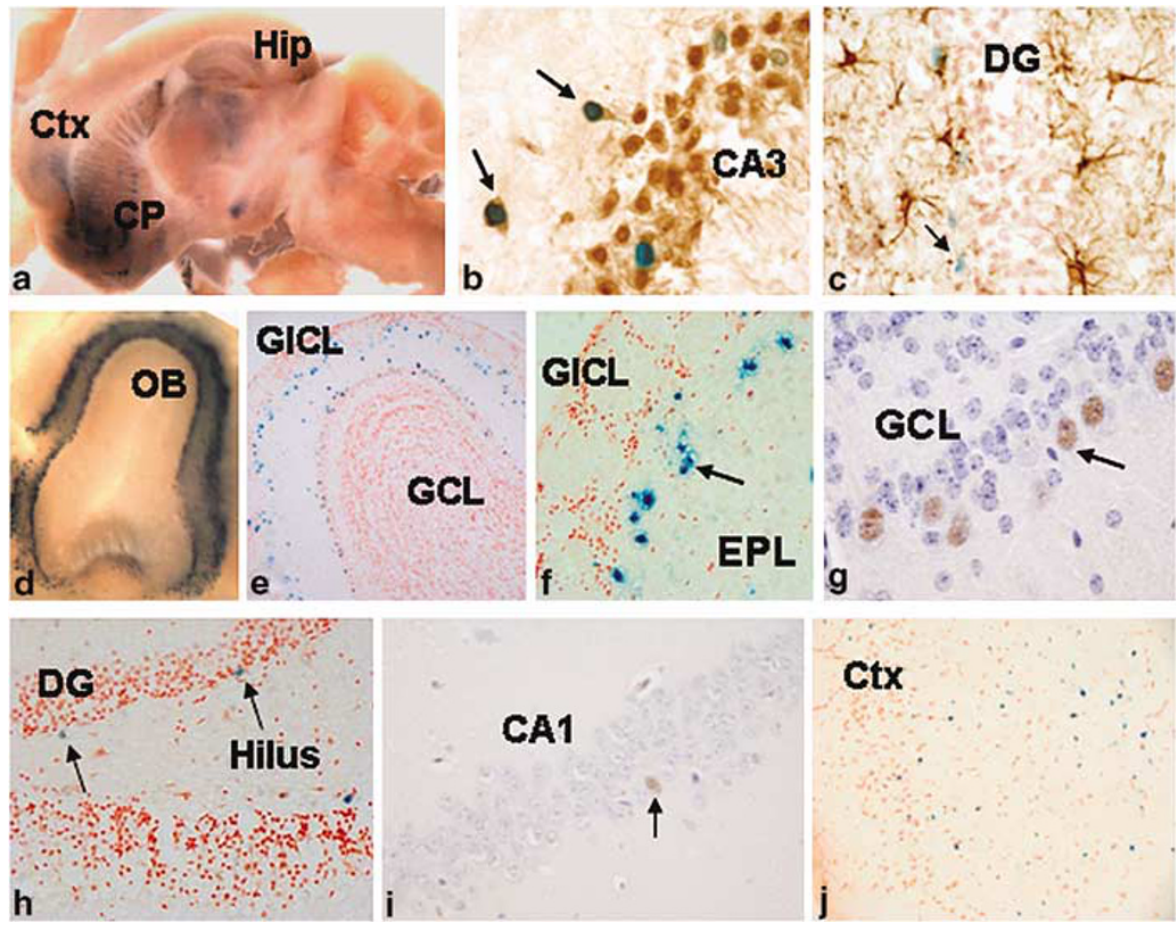

Figure 3 Expression of ALK message as detected by knocked-in $\beta$-gal detection, by immunoreactivity ( $g$ and $\mathrm{i}$ ) or enzymatic detection using $\mathrm{X}$-gal as a substrate (a-f, h, and j). (a) Broad distribution of $\beta$-gal-expressing cells in the forebrain of ALK HE animals; notice strong expression in frontal cortex (Ctx), caudate putamen (CP), hippocampus (Hip), and various thalamic and subthalamic nuclei; $(b-c)$ double staining showing $X$-gal substrate reactivity and immunodetection for neuronal (NeuN) or Glial (GFAP) expression in various hippocampal regions shows that $\beta$-gal activity is detected exclusively in mature neurons. Similar results are observed in other brain regions. ( $d-f$ ) Prominent expression is detected in olfactory bulbs; (d) whole mount $\mathrm{X}$-gal detection; (e-g) display strong expression olfactory bulb, including expression in a subset of presumed tufted cells in the external plexiform layer (EPL, arrow) surrounding the glomerular $(\mathrm{GlCL})$ cell layer (f). In (g) arrow points to prominent immunoreactivity in a subset of mitral cells bordering the granule cell layer $(\mathrm{GCL})$ in the inner plexiform layer. (h-i) Expression in the hippocampus shows a small subset of mature neurons detected in the CAI-CA3 layers and in the DG of the hippocampus, typically localized in the SGZ (DG); in no cases did we observe colocalization with BrdU in dividing neuronal progenitor cells (data not shown); (j) shows scattered neurons expressing ALK transcripts in the frontal cortex. 


\section{Age-Dependent Increase in Baseline Hippocampal Progenitor Proliferation in ALK Mutant Mice}

Based on the brain expression of the ALK transcripts during development and in the adult (this work; Iwahara et al, 1997; Morris et al, 1997), and the role of the putative ALK ligands midkine and pleiotrophin in hippocampal synaptic plasticity and neuronal development (Amet et al, 2001; Nakamura et al, 1998), we analyzed the ALK HO specifically for changes in hippocampal morphology and baseline proliferation as part of a larger effort utilizing changes in hippocampal neurogenesis to identify novel targets. Overall, hippocampal morphology appeared intact in 6-month-old ALK HOs. We labelled progenitor cells in vivo with BrdU $(100 \mathrm{mg} / \mathrm{kg}$ i.p. for four consecutive days) and analyzed hippocampal proliferation of progenitor cells by FACS (Bilsland et al, 2006) and immunohistochemistry. This analysis revealed a statistically significant increase in the number of BrdU-positive cells in the hippocampus of ALK mutant animals, compared to WT littermates $(n=8$ animals/group; Figure 4). This finding was verified by conventional immunohistochemistry quantification of BrdU and PCNA IR nuclei in the SGZ of the DG (Figure 4a-d). In both cases, there was a significant increase in progenitor cells in the DG of ALK mutant animals. To assess whether the enhanced proliferation in the hippocampus gave rise to increased number of DG newborn neurons, we analyzed the expression of doublecortin, a marker of committed neuroblasts (Rao and Shetty, 2004; Brown et al, 2003) and calretinin, a postmitotic immature marker for DG neurons
(Kempermann et al, 2004b). In both cases, we detected an increased expression of IR cells in the DG of ALK mutant mice, consistent with increased neurogenesis in these animals (Figure 4). Although the increased number of DCX-positive cells failed to reach significance, the increased numbers of calretinin IR cells was significant. The increased immunoreactivity was restricted to the SGZ of the DG (Figure $4 \mathrm{e}-\mathrm{g}$ ), although we observed no differences in the numbers of calretinin-positive GABAergic interneurons in the hilus or in the CA1 layer of the hippocampus (Figure $4 \mathrm{n}$ and data not shown), populations that do not arise from adult progenitor cells. This was verified by direct quantification of the calretinin-positive hilar cells (Figure 4n). Therefore, the enhanced number of calretinin-positive cells can be attributed to an increase in basal proliferation in the hippocampus following loss of ALK activity. In addition, we also monitored the numbers of calbindin and parvalbumin IR neurons in the hippocampus and observed no change in the ALK KO animals (data not shown). However, the changes observed in older animals (6-8 months) in baseline proliferation were not observed in animals evaluated at 2-3 months of age (Figure 5 and data not shown) in two independent experiments. Following a FACS evaluation of younger animals, we simultaneously monitored both the degree of proliferation and survival of labelled progenitors in younger animals with a modified protocol using two distinct halogenated thymidine analogs (Figure 5; Vega and Peterson, 2005). In this paradigm, mice were given $50 \mathrm{mg} / \mathrm{kg}$ IdU for two consecutive days on days 1-2 (to label the progeny from cells dividing at the beginning of the study)

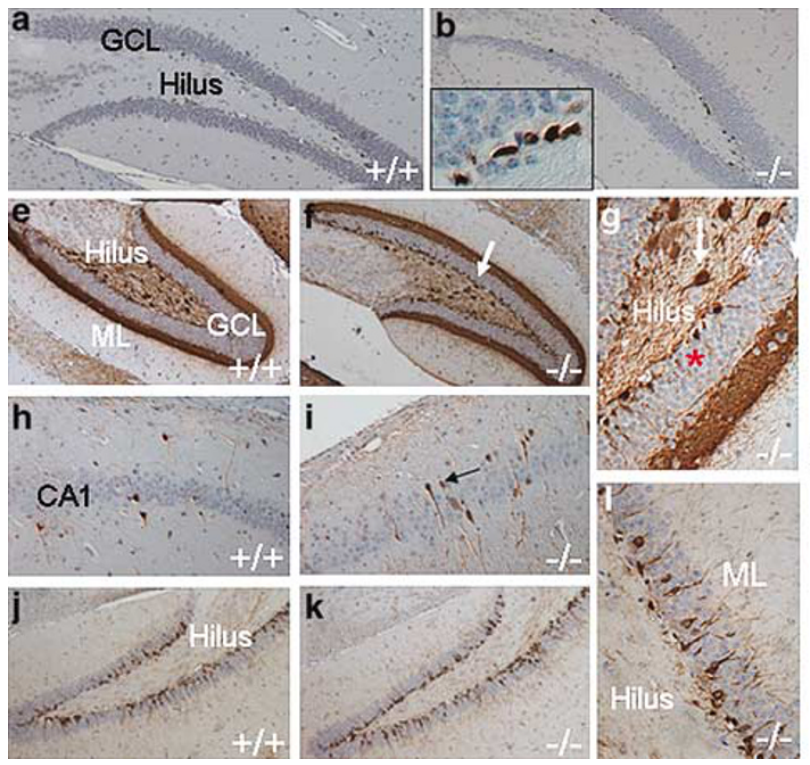

c

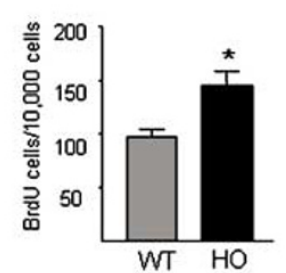

m

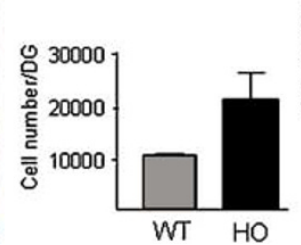

d

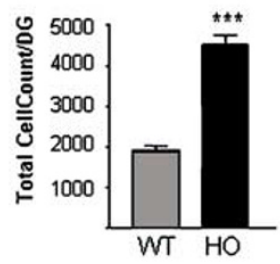

n

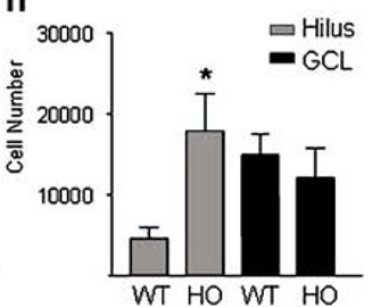

Figure 4 Quantification of neural progenitor proliferation in the DG of ALK HO vs WT littermates. (a-b) BrdU immunoreactivity in the hippocampus in WT littermates (a) and in the ALK HO animals (b). Inset displays higher magnification of the region. Animals were dosed with $100 \mathrm{mg} / \mathrm{kg}$ i.p. of BrdU for four consecutive days and then processed for immunohistochemistry and FACS quantification. (c) FACS analysis of BrdU quantification in WT and ALK HO animals. (d) Quantification of PCNA IR nuclei in the DG of WT and HO animals. Data is expressed as extrapolated total number of cells per hippocampus. Expression of calretinin (e-i) and doublecortin ( $k-j)$. Calretinin expression in WT (e) and ALK HO animals (f) shows differences in SGZ immunoreactivity. Arrow in ( $f$ ) points to the SGZ expression of calretinin-positive postmitotic newborn neurons in the granule cell layer (GCL). (g) A larger image of ( $f$ ). Arrow points to the hilar mossy cells, which are also calretinin IR, although these are GABAergic interneurons. Asterisk shows a calretinin-positive newborn neuron in the GCL. (h-i) By contrast, calretinin expression in the WT CAI (h) and ALK HO (i) shows no overall change in IR cell numbers. (j-k) Doublecortin immunoreactivity in the hippocampus of WT (j) and ALK HO (k). (I) A higher magnification inset of (k). (m-n) Quantification of IR cells in WT and ALK HO animals for $(m)$ doublecortin and $(n)$ calretinin. Note increase in number of calretinin-positive cells in the granule cell layer (GCL) but not in the hilar mossy cell counts ( $n=5$ animals/group). ML, molecular layer of the DG; GCL, granule cell layer. 

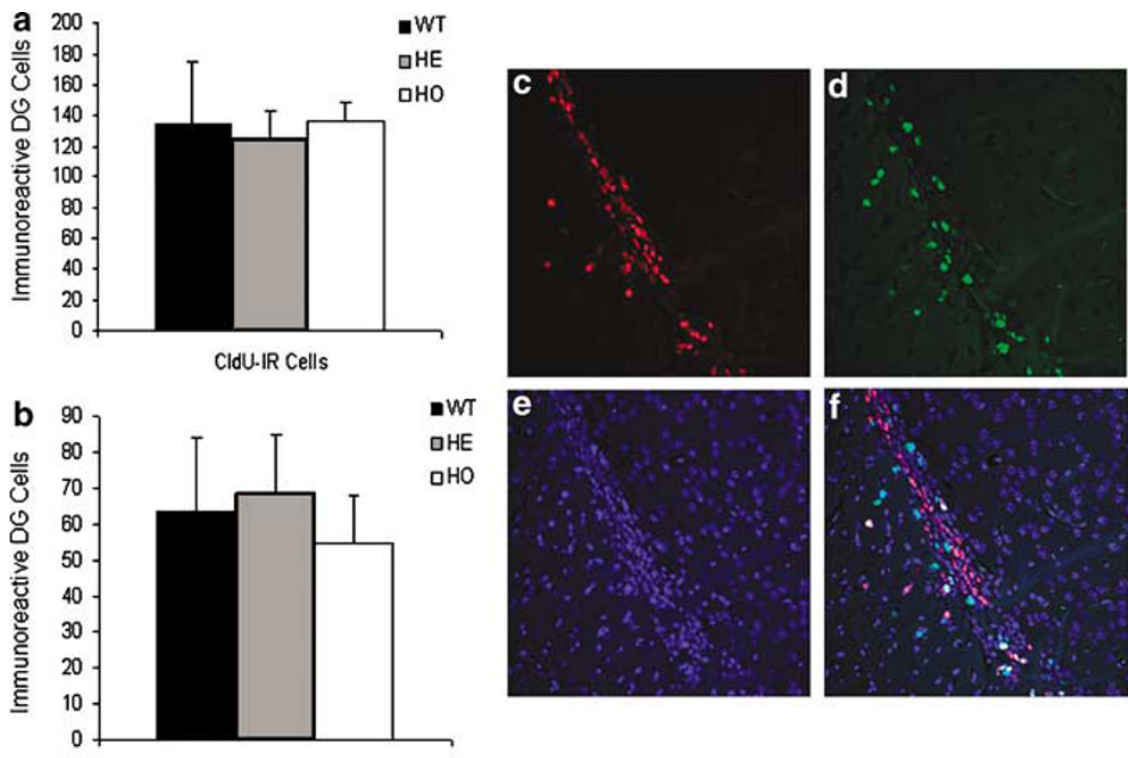

IdU-IR Cells

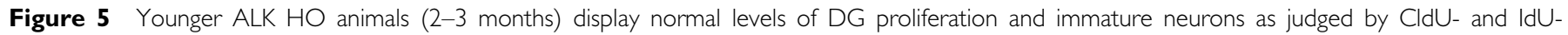
incorporation studies. IdU was administered 2 weeks before the end of the study and reflects survival of newborn cells. CldU was administered during the

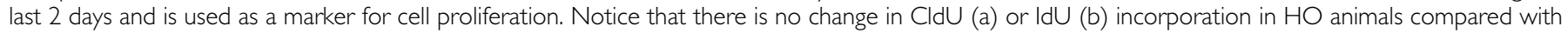
HE (HEs) or WT animals. Notice that approximately $40 \%$ fewer cells were labelled with IdU than CldU, accounting for significant lack of survival of newborn

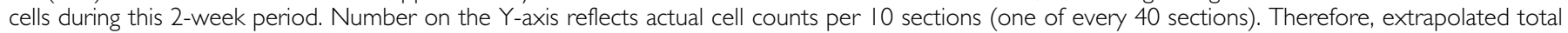

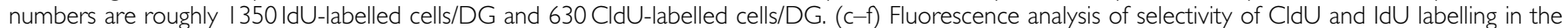

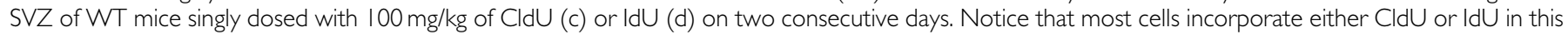
paradigm. (e) TOTO3 staining and a merged image of TOTO3, CldU and IdU is shown in (f), depicting the specificity of the immunodetection protocol employed here.

and CldU (50 mg/kg) was dosed during days $12-13$, to label dividing cells one day before killing the animals. We then quantified both the numbers of CldU- and IdU-positive cells in the DG of these animals. Results from this experiment demonstrate that approximately $47 \%$ fewer cells were labelled with IdU, suggesting that a significant proportion of newborn cells did not survive after 2 weeks, consistent with previous reports (Figure $5 \mathrm{a}$ and $\mathrm{b}$ ). The extrapolated total numbers are roughly 1350 IdU-labelled cells/DG and 630 CldU-labelled cells per WT DG in this experimental paradigm. The specificity of the immunoreactivity for CldU and IdU analogs can be seen in an additional experiment where animals were dosed with each analogue on two consecutive days (Figure $5 \mathrm{c}-\mathrm{f}$ ), as judged by the distinct pattern of labelling of dividing cells in the ventricular walls. Overall, we found no differences in the total numbers of labelled cells in the DGs of the ALK HO and WT animals, suggesting an age-dependent effect of ALK disruption in the regulation of hippocampal progenitor proliferation. This finding raised the possibility that ALK might not function cell-autonomously in the regulation of hippocampal proliferation and that developmental alterations are unlikely to explain the increased proliferation observed in the older animals.

\section{Analysis of SVZ Stem Cell Biology in the ALK HO Animals}

Owing to the results obtained with the hippocampal progenitors in the older $\mathrm{HO}$ animals, we wanted to test whether the long-term repopulating stem cells located in the adult ventricular walls also displayed cell-autonomous alterations in proliferation. Towards this goal, we isolated SVZ preparations of stem/progenitor cells from $n=6$ individual mice from each genotype, as described in Bonnert et al (2006). Individual animal dissociations were carried out to ensure reproducibility across each genotype. From this analysis, we observed that ALK HO and WT animals generated similar numbers of primary neurospheres (Figure 6), suggesting that the number of SVZ progenitor/stem cells were unaltered. To evaluate the number of stem cells, primary neurospheres were dissociated and cultured in clonal conditions $(1 \mathrm{cell} / \mu \mathrm{l})$ in standard medium containing FGF2 and EGF. The average number of secondary spheres, although variable across individual animals, did not reveal any differences across genotype (Figure 6b). We also assessed cells grown clonally (from secondary sphere dissociates) in various growth factor concentrations (Figure 6c) and measured proliferation rates in the presence of FGF2, EGF, or both, using an ATP-bioluminescence assay. This analysis again revealed no changes across genotypes. We were also unable to detect long-term effects after six passages in culture, suggesting that the loss of function of ALK does not affect SVZ stem cell and progenitor proliferation.

\section{Behavioral Analysis of ALK Mutant Mice}

The 'neurogenesis hypothesis' stipulates that an increase in baseline hippocampal neurogenesis will lead to an improvement in a subset of hippocampal-dependent functions (Kempermann 2002; Kempermann et al, 2004a) and 

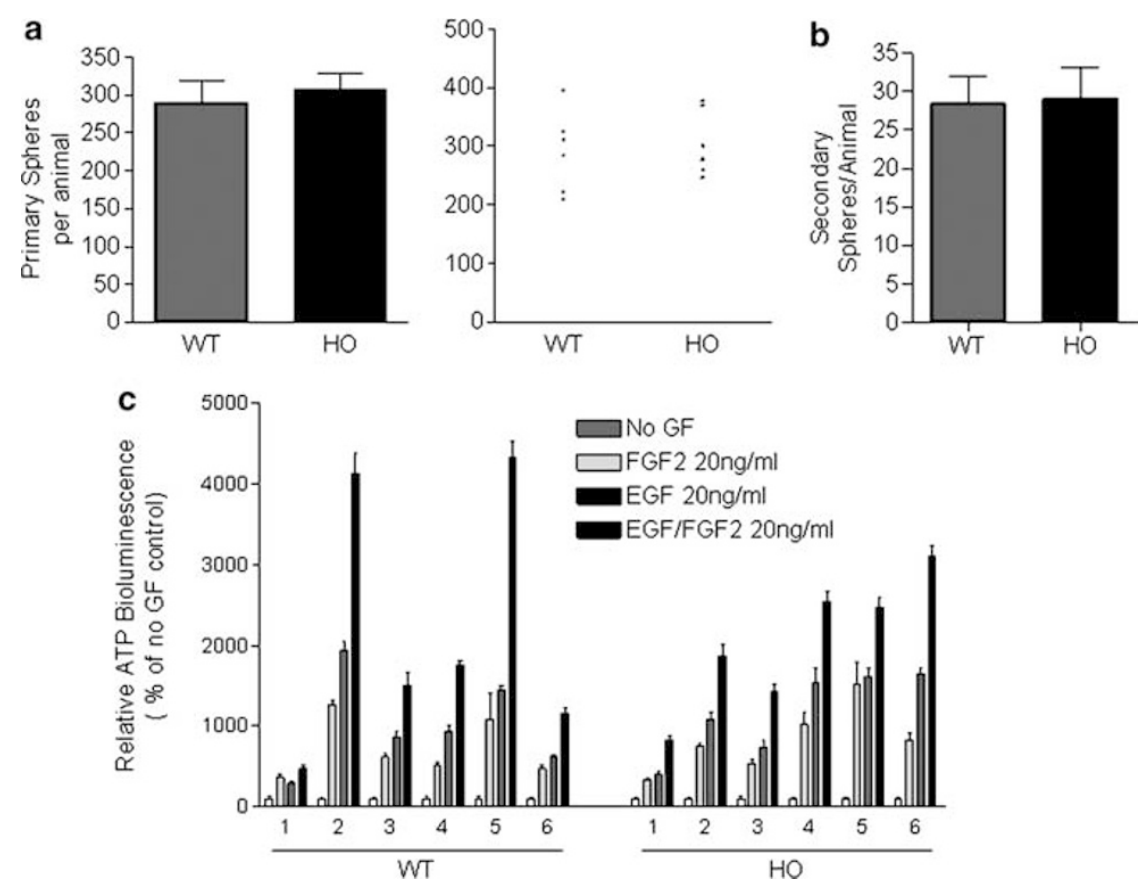

Figure 6 Evaluation of ALK involvement in stem cell modulation of adult SVZ stem and progenitor cells. Individual SVZ dissociates obtained from either WT or $\mathrm{HO}$ mutant animals ( $n=6$ per genotype) were plated and grown for 8 days in normal growth medium containing FGF2 and EGF. Individual spheres per animal were counted (a-b). Secondary spheres were obtained following dissociation of primary spheres and grown clonally in 96-well plates (c). We observed no differences in both instances. From the third passage, cells were dissociated and cultured for 3 days in various growth factor backgrounds under clonal conditions. Total cell numbers were obtained by measuring ATP content. In no instances did we observe a difference between WT and HO cells expanded in vitro.

potentially to an antidepressant profile (Santarelli et al, 2003). In our strategy, we used basal hippocampal proliferation as an in vivo readout of hippocampal activity, aimed at identifying new targets. To evaluate whether the loss of ALK function altered behavior or hippocampal function, we tested the ALK mutant mice in tests typically used to monitor efficacy of mood-modulating psychoactive molecules as well as in a cognitive test dependent on intact hippocampal circuitry. Initially, we tested the animals in the TST and the PST, tests sensitive to classical monoaminergic modulation (Cryan and Mombereau, 2004; Mori et al, 2005; Ferrari and Giuliani, 1997). Both of these tests are sensitive to acute administration of existing antidepressant compounds, which act by modulating both serotonergic and adrenergic transmission (Cryan and Mombereau, 2004). In the TST, animals are suspended from the tail for a 5-min interval and the struggle time is measured. Classical antidepressants, when applied acutely, decrease the immobile time, due to the enhanced struggle time of the animals. In the PST, the struggle time is measured when animals are lowered into containers filled with water. To rule out a potential confounding effect of basal locomotor activity in the ALK mice, we monitored overall mobility scores in both cohorts and showed that locomotor activity was not different in the ALK and WT littermates (Figure 7a). No difference was seen between male and female immobility scores for the WT animals in the TST. The data from both sexes could therefore be combined and used in the final analysis. In these tests, the ALK mutant mice demonstrated a significant decrease in immobility when compared with the WTs $(p<0.03$; Figure $7 c)$. There was a slight but significant difference in body weights between ALK mutant and WT mice. However, when mice were weight matched for each group $(n=6)$, the significant difference in immobility scores between ALK mutant and WT mice was even more apparent $(p<0.007$; data not shown). An essentially identical result was observed in the PST (Figure 7d) in which the animals spent significantly less time immobile. In addition, we evaluated the ALK HO animals in a classical anxiety paradigm, the black/white box test. In this test, the amount of time animals spend in the light and dark areas of the box is measured as well as the numbers of crossings between the dark and light areas. Anxiolytic compounds typically result in an increased percentage of time being spent in the light portion of the box and anxiogenic compounds lead the animals to spend most of their time in the dark compartment. In this test, we observed no difference between ALK HOs and their littermates (Figure $7 \mathrm{~b}$ ). Altogether, these data suggest that ALK mice display an antidepressant profile that cannot be explained by a change in locomotor activity or anxiogenic effect of loss of ALK activity.

\section{Assessment of Hippocampal Function in a NOR/NOL Paradigm}

In addition, we wanted to test for hippocampal function in the ALK mutant animals, by measuring their performance in hippocampal-dependent tasks that have been associated with changes in newborn neuron production and that are dependent on an intact hippocampal circuitry (Shors et al, 2001; Bruel-Jungerman et al, 2005; Snyder et al, 2005). 
a Locomotor Activity

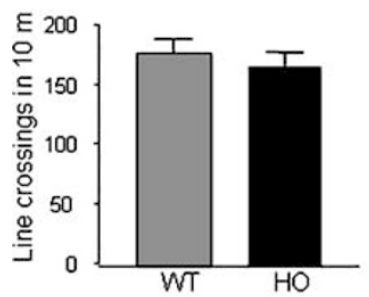

c Tail Suspension Test

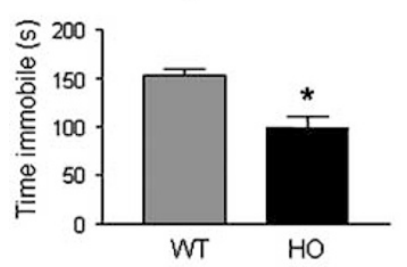

d Porsolt Swim Test

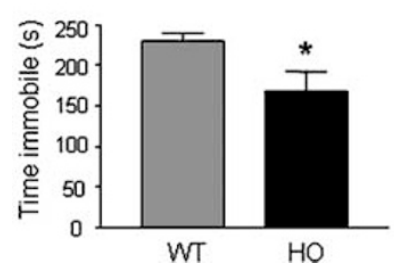

Figure 7 Antidepressant behavior of ALK KO animals. Behavior of WT $(n=8-10)$ and ALK HOs $(n=10)$ in the locomotor activity test (a), the black/white box anxiety test (b), the TST (c) and in the Swim Porsolt Test (d). A separate cohort of animals was used for the black/white anxiety test ( $n=10$ animals each group). In (c-d), behavioral performance is plotted as the average time immobile within the 5 min study (left graph) and as a scatter plot graph of individual animals within the study (right). ALK KO mice display a decrease time in both tests, a profile typical of acute antidepressant exposure. Notice in $(a-b)$ there is no difference between the two groups in basal locomotor activity or in an anxiety test, as measured by time spent in black section of the platform.

Therefore, we tested the animals in the NOR/NOL test, which essentially measures the ability of animals to habituate to a familiar environment and recognize either novel objects placed in their environment (novel object recognition) and novel spatial distribution of familiar objects (in the novel-object location task, a measure of hippocampal performance). In brief, animals are trained to familiarize themselves with a variety of objects ( $n=5$ objects) placed during the training period (see Methods). After four training sessions, animals are exposed to rearranged familiar objects (in session 5) or to a new object (in session 7). Typically, animals exposed to a new object or an object placed in a new location spent more time exploring these objects $v s$ familiar objects. The measure of the time spent on novel $v s$ familiar objects is scored as $\mathrm{d} 1$, a reflection of the animals' ability to remember the location and familiarity with these objects. In our hands, we observed no significant difference between the $\mathrm{HO}$ and WT mice in their levels of object exploration during the three-sample sessions, suggesting no overall differences in activity. In the NOL session, both $\mathrm{HO}$ and WT mice spent significantly more time exploring the novel/switched objects, as shown by the significant increase in the $\mathrm{d} 1$ scores over those scored in the pre-NOL session (Figure 8). Similarly, both groups spent more time exploring the novel object in the NOR session than in the pre-NOR session, suggesting no hippocampal deficits. However, HO mice also scored a significantly higher $\mathrm{d} 1$ score than the WT mice in the NOR session, indicating that the $\mathrm{HO}$ mice explored the novel object more than their WT counterparts. Although there was a trend for an increased $\mathrm{d} 1$ score in the NOL, this was minimal and did not reach significance (due to the higher exploratory activity before the training sessions in the ALK HO mice than the WT in the pre-NOL period; Figure $8 \mathrm{c}$ and $\mathrm{d}$ ). These results demonstrate, most importantly, that the increase in baseline neurogenesis does not reflect a compensatory mechanism that might have ensued from a hippocampal deficiency, which would be detected in these hippocampaldependent tasks. Thus far, the data obtained suggest a potential beneficial effect of ALK loss of function in cognition, although further tests are required to assess specific effects on various cognitive domains that rely on frontal cortex or hippocampal circuitry.

\section{Neurochemical Analysis of Extracellular Dopamine and Serotonin Systems Reveals Alterations in Prefrontal Cortex Dopamine Metabolism}

The widespread expression of ALK in the adult brain, as judged by transcript expression (Iwahara et al, 1997) and the pattern of $\beta$-gal in the knock-in mice described here suggest that a variety of circuits implicated in the behavioral alterations observed could be differentially modulated in the KO animals. The changes initially detected in progenitor proliferation in the hippocampus cannot be due to a cellautonomous effect of the loss of ALK function, based on the expression pattern of $\beta$-gal as well as due to the fact that stem cells derived from the ALK HO mice display identical properties as those derived from WT littermates. Therefore, to assess whether critical neurotransmitter pathways known to regulate both hippocampal neurogenesis as well as affect the performance in the depression and cognitive tests described here, we monitored the extracellular levels of dopamine, serotonin, and their metabolites in dissected prefrontal cortex, striatum, and hippocampus of 2-5month-old animals before the changes observed in the hippocampal progenitors. (Figure 9). The overall levels of serotonin (5HT) and the ratios of serotonin/5HIAA (5-hydroxyindoleacetic acid) were not significantly different in the frontal cortex or striatum, but there was a significant decrease in the extracellular levels of serotonin in the hippocampus in the KO animals (Figure 9b and d). However, the 5HT metabolite 5-HIAA levels did not decrease significantly and therefore the decrease in hippocampal extracellular serotonin is probably not biologically significant. This aspect will require further investigation.

We also monitored dopaminergic tone in the prefrontal cortex and striatum by measuring extracellular levels of dopamine and two metabolites (Figure 9a and c). We did not measure hippocampal dopamine tone due to the lack of sensitivity of this methodology in this region. In contrast to serotonin, levels of extracellular dopamine content (with an average change of $454 \%$ levels of extracellular dopamine 

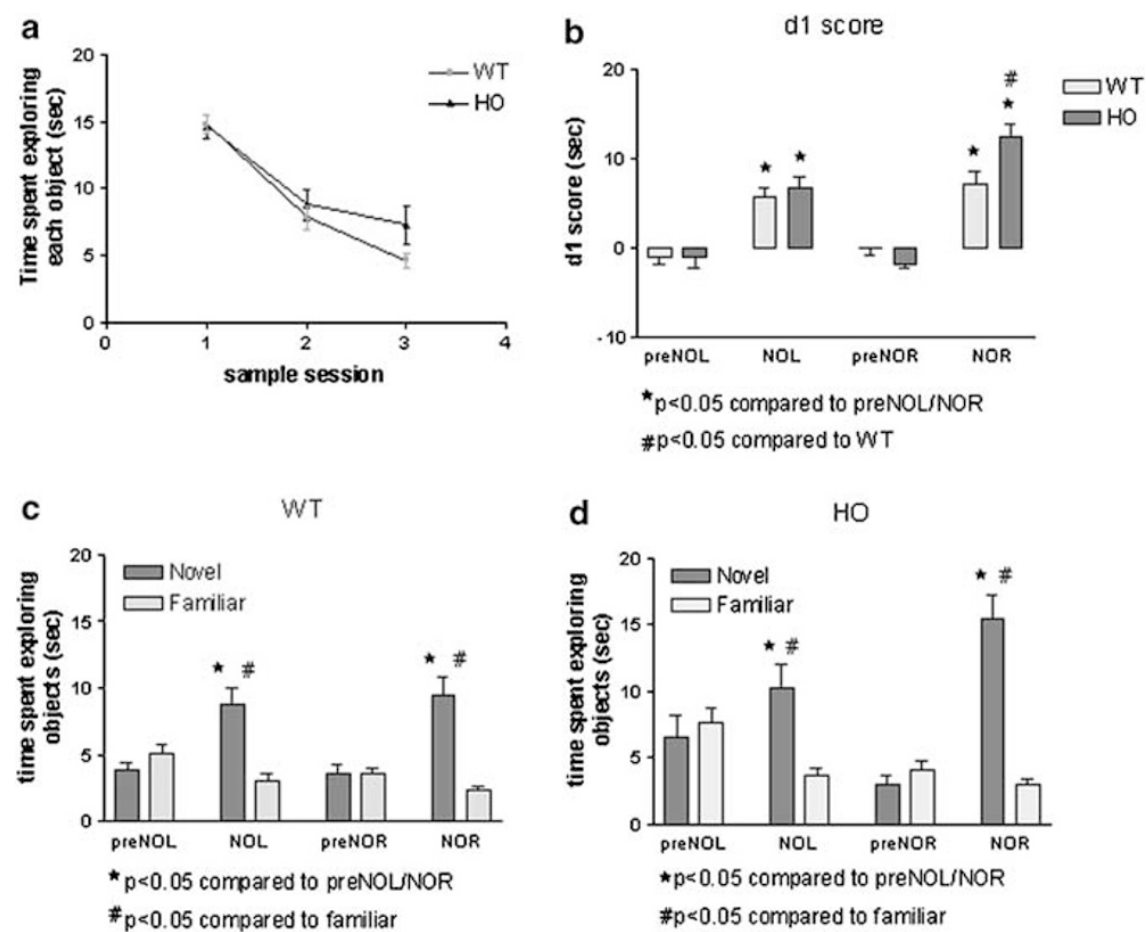

Figure 8 Analysis of hippocampal performance in a NOR/NOL test. (a) Analysis of exploration time. Differences between both genotype groups are not significant. (b-d) Analysis of time spent exploring novel objects and novel locations. (b) Measure of dl scores. ALK HO animals display enhanced performance selectively in the NOL test compared to their WT littermates. Both groups display statistically significant effects in remembering novel objects and locations (c-d) Both WT and ALK KO animals are able to learn to recognize familiar and novel objects and their respective locations in the platform, highlighting normal hippocampal spatial and cognitive functions; this finding suggests that the increased basal neurogenesis does not result from or leads to an impairment of hippocampal function. $\mathrm{dl}$, time exploring novel object-time exploring familiar objects. All scores are averaged by number of objects.

compared with the WT animals) and its metabolites DOPAC and HVA in the prefrontal cortex, but not the striatum, were significantly increased in the ALK HO animals (133\% in DOPAC levels and $160 \%$ over WT levels in the case of extracellular HVA). The concomitant changes in extracellular dopamine and both metabolites strongly suggest that the basal tone of dopamine signalling in the prefrontal cortex is increased in the ALK mutant mice. By contrast, we observed a decrease in dopamine levels in the striatum of the mutant mice, although the lack of variation in both dopamine metabolites argues for the lack of a biological effect of dopaminergic tone in the striatum. This result is consistent with the lack of locomotor alterations observed in the behavioral paradigms reported here. The changes observed support a role for ALK signalling in a selective modulation of mesocortical dopaminergic synaptic transmission. Whether similar changes in dopaminergic tone occur in the hippocampus, which could explain the neurogenic changes described, remains to be determined. Alternatively, since increased dopaminergic signalling in the prefrontal cortex has been associated with an enhancement of cognitive function and antidepressant efficacy, it is plausible that the alterations in proliferation in the hippocampus might be an indirect effect of synaptic function in cortical areas. The regulation of dopamine signalling in the frontal cortex constitutes an important area of research for schizophrenia and depression and might explain the behavioral changes described in this report (Siever and Davis, 2004; Yang and Chen, 2005).

\section{DISCUSSION}

The regulation of hippocampal neurogenesis has received considerable attention in recent years due to the correlation between changes in baseline proliferation and the physiological effects that ensue following neuroendocrine and monoaminergic modulation in rodents and primates (Lie et al, 2004; Kempermann et al, 2004a). This has been particularly suggestive of an involvement of hippocampal neurogenesis in the regulation of mood, probably through the modulation of hippocampal function and its relationship with the hypothalamus (Kempermann et al, 2004a). The lack of efficacy of antidepressant administration in a few murine models where proliferation was disrupted by Xirradiation or the addition of antimitotic agents (Santarelli et al, 2003; Monje et al, 2003; Bruel-Jungerman et al, 2005) have led to the hypothesis for the requirement of hippocampal neurogenesis in antidepressant efficacy. In addition, hippocampal neurogenesis has been postulated to play a role in several aspects of learning and memory, similar to the functional involvement of new neuron birth in songbirds (Louissaint et al, 2002). Existing data suggest that changes in the baseline of hippocampal neurogenesis can act as an in vivo biomarker for central effects of psychoactive molecules and might be utilized as an assay to identify new molecular components with an effect in adult CNS function. As part of a larger evaluation of various mutant lines, we identified ALK as a locus that affected the number of progenitor cells in the adult hippocampus. This 

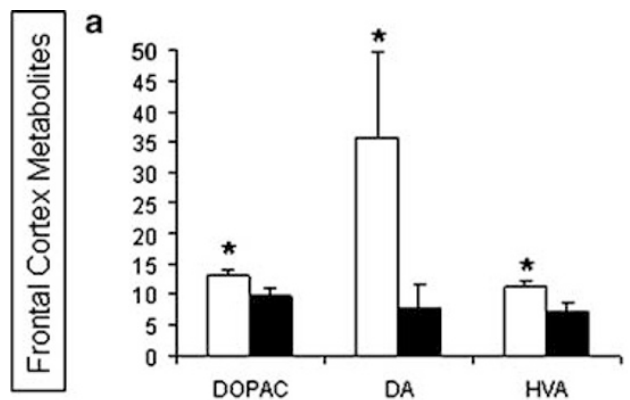

b
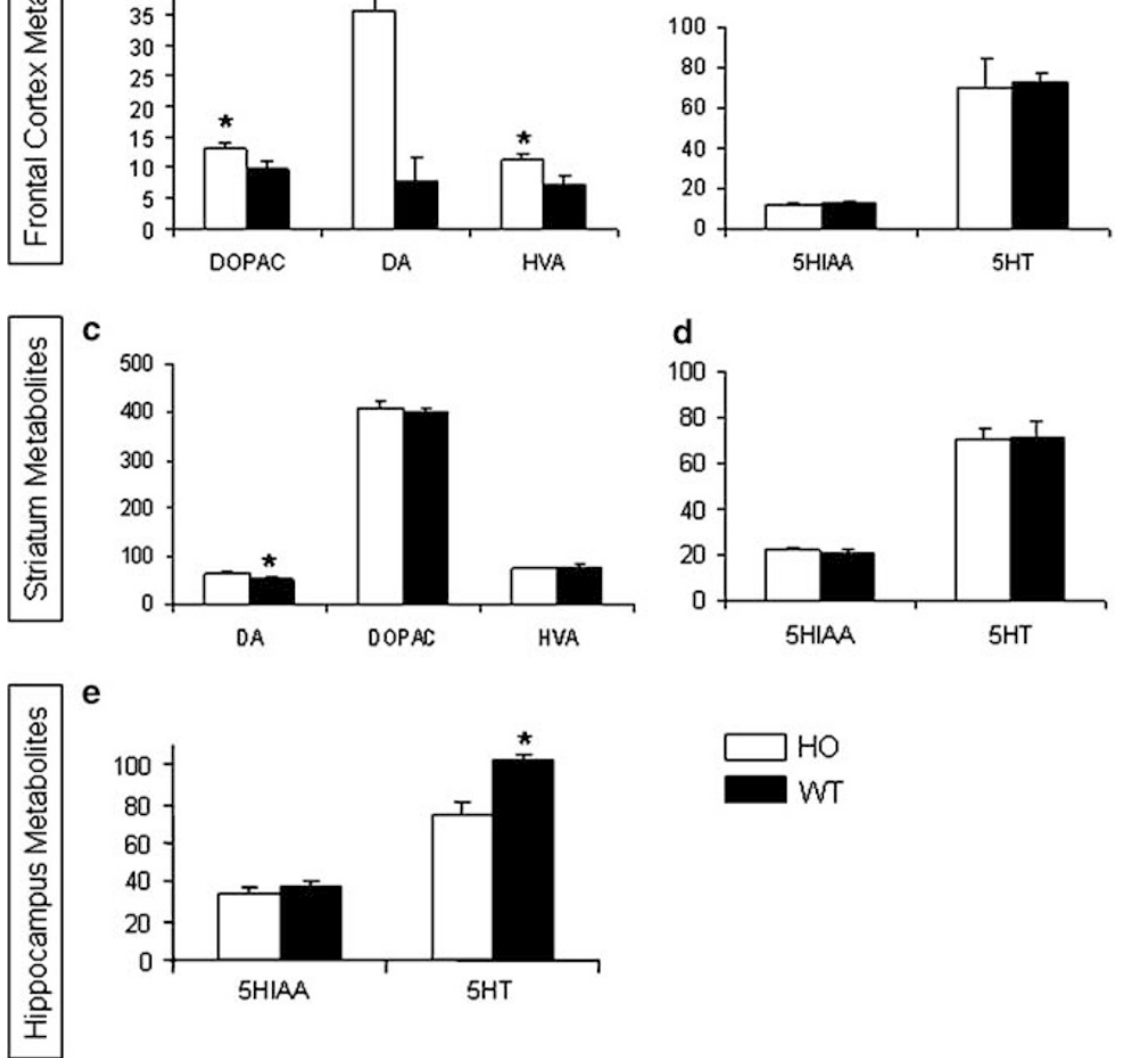

Figure 9 Neurochemical analysis of serotonin, dopamine, and metabolites in the frontal cortex, striatum, and hippocampus of ALK KO mice. Data are plotted as ng/g of wet weight tissue and expressed as mean \pm SEM. Notice the statistically significant increases in the extracellular levels of dopamine and its metabolites DOPAC and HVA selectively in the frontal cortex the ALK HO animals compared with WT levels. In the striatum, dopamine levels are decreased, although levels of the metabolites are unaltered, implying normal dopamine tone in this region. Extracellular hippocampal serotonin levels appear selectively decreased in the ALK KO animals, although levels of its metabolite 5HIAA are unchanged and therefore presumed to be of little biological significance.

effect was observed in the absence of obvious morphological abnormalities in the brain or any evidence of a gross developmental alteration. This is evidenced by the unaltered pattern of several neuronal populations in the hippocampus, as judged by the expression of the calcium-binding proteins calbindin, parvalbumin, and calretinin immunohistochemistry.

Although ALK function has been associated with cellcycle regulation in a variety of tumor cell lines, both as a full-length receptor as well as in the frequent ALK-NPM fusions ensuing from chromosomal translocations in a variety of lymphomas (Coluccia et al, 2005; OsajimaHakomori et al, 2005), the endogeneous role of ALK has remained uncertain in either the hematopoietic system or in the nervous system. Among the ascribed ligands for ALK activation, both midkine and pleiotrophin are expressed and regulated in neural progenitors (Winkler et al, 2002; Bonnert et al 2006; Furuta et al, 2004; Jung et al, 2004). Specifically, the loss of pleiotrophin leads to alterations in the threshold for long-term potentiation (LTP) in CA3-CA1 synapses (Amet et al, 2001; Pavlov et al, 2002) although the physiological consequences of this change or the effects in progenitor cell number were not analyzed in this study. In acute hippocampal preparations from the ALK mutant mice, we observed no alterations in basal activity, LTP threshold, or maximal response after a theta burst paradigm (data not shown), strongly arguing that there are no deficits in glutamatergic transmission in the adult hippocampus of ALK HO animals, in contrast with the neurochemical alterations described for the monoaminergic pathways. Mice deficient in midkine function display deficits in cognitive performance and an anxiogenic phenotype, which are not observed in the ALK HO mice (Nakamura et al, 1998). Additionally, in the midkine KO mouse, Nakamura et al (1998) described a temporal 'maintenance' of calretinin immunoreactivity in the hippocampus. On close examination of the data, it is apparent that the changes in calretinin immunoreactivity are restricted to the SGZ, and not the hilar mossy cells, lending support to the notion that the changes observed in the midkine $\mathrm{KO}$ might be due to alterations in progenitor cell number. The temporal nature of the effects reported in this mouse line, similar to what we observed in the ALK HO animals, might be explained by a change in the contribution of ligand-dependent ALK activation in the adult, where additional ligands might act in a redundant manner to control progenitor numbers in 
the hippocampus. To this complexity must be added the involvement of additional receptors for midkine and pleiotrophin, the membrane tyrosine phosphatase PTP-zeta (Meng et al, 2000; Maeda et al, 1999; Sakaguchi et al, 2003) and syndecan-3, which are highly expressed in the adult CNS, including neurogenic regions (Bonnert et al, 2006; Winkler et al, 2002). To assess a potential contribution of these molecules to the phenotypes reported here, we measured mRNA levels of syndecan-3, pleiotrophin, midkine, and PTP-zeta by qPCR in various brain regions of the KO animals and detected no changes (as shown in Figure $2 b$ ), suggesting that the behavioral effects observed cannot be due to a loss of expression of these molecules.

However, in support for the potential involvement of these pathways in dopaminergic signalling, a previous report identified the ALK ligand pleiotrophin as a differentially regulated transcript in the striatum of mice in response to a loss of afferent innervation following 6-OHDA lesions (Hida et al, 2003). In this report, through a microarray analysis, pleiotrophin was the highest differentially regulated gene in the striatum, suggesting that signalling mediated by pathways activated by this ligand might be implicated in the responsiveness to dopamine signalling. Exposure of dopaminergic neurons to pleiotrophin enhanced their survival in vitro (Hida et al, 2003). Therefore, the loss of ALK might affect the synaptic properties of dopaminergic cells. An exact investigation on the mechanistic links between ALK signalling in various dopaminergic populations remains to be determined. The broad expression of ALK in the adult brain, as judged by previous reports and the $\beta$-gal staining reported here, includes areas containing dopamine-producing cells and neurons innervated by dopaminergic cells, including the frontal cortex, striatum, substantia nigra, hippocampus, and the periglomerular cells of the olfactory bulb. Whether the loss of ALK expression affects some of these populations differentially requires further characterization and is beyond the scope of this initial report.

The dopamine hypothesis of schizophrenia stems from the fact that clinically efficacious antipsychotics have the ability to inhibit dopamine D2, D3, and D4 receptors (Yang and Chen, 2005; Siever and Davis, 2004). Schizophrenia patients who have received antipsychotic treatment show an increase in the dopamine metabolite HVA in prefrontal and cingulated cortices, similar to the phenotype observed here in the ALK loss of function. This, together with the behavioral effects observed in the ALK mice, the 'antidepressant' profile and enhanced performance in the novel object-recognition test, suggest that modulation of ALK function can have important implications in behavior and neural physiology. However, a more detailed investigation of the changes observed in dopamine signalling remains to be conducted, through measurements of synaptically released dopamine and in experimental paradigms manipulating the mesocortical dopaminergic circuitry through pharmacological modulation. The variable changes in hippocampal progenitor proliferation observed are potentially a consequence of the alterations in prefrontal cortex function and potentially reflect an alteration in dopaminergic innervation of the hippocampus, although this remains to be determined. In any case, ALK loss of function cannot explain a direct effect on progenitor proliferation, as judged by the expression pattern in the hippocampus and lack of effect in adult stem cell biology. However, the increased baseline proliferation is consistent with the antidepressant profile of these animals, and the changes observed in the novel object recognition, a measure of contextual learning dependent on the hippocampus and correlated with changes in hippocampal proliferation (Bruel-Jungerman et al, 2005; Shors et al, 2001).

Interestingly, a recent report has identified a couple of nonsynonymous polymorphisms in the ALK locus that segregate with some schizophrenia families (Kunugi et al, 2006). The data presented here are consistent with a potential role for ALK-mediated signalling in mesocortical dopaminergic circuitry development or function and defines ALK as a potential target for schizophrenia. Currently, several ALK-specific inhibitors are being developed and tested in the clinic for the treatment of a variety of lymphomas and other cancers where ALK activity might be critical in neoplastic transformation. The application of these small molecules to adult CNS disorders will undoubtedly shed light on these questions and will pave the way for understanding whether a pharmacological antagonism of ALK function will yield neurophysiological effects similar to the genetic loss of function reported here.

\section{CONFLICT OF INTEREST}

We declare that, except for income received from our primary employer, no financial support or compensation has been received from any individual or corporate entity over the past 3 years for research or professional service and there are no personal financial holdings that could be perceived as constituting a potential conflict of interest.

\section{REFERENCES}

Amet LE, Lauri SE, Hienola A, Croll SD, Lu Y, Levorse JM et al (2001). Enhanced hippocampal long-term potentiation in mice lacking heparin-binding growth-associated molecule. Mol Cell Neurosci 17: 1014-1024.

Bilsland JG, Haldon C, Oliver KR, Murray F, Wheeldon A, Wainwright A et al (2006). A rapid method for the quantification of mouse hippocampal neurogenesis in vivo by flow cytometry; validation with conventional immunohistochemical methods. J Neurosci Methods 157: 54-63.

Bonnert TP, Bilsland JG, Guest PC, Heavens R, McLaren D, Dale C et al (2006). Molecular characterization of adult mouse subventricular zone progenitor cells during the onset of differentiation. Eur J Neuroscience 24: 661-675.

Brown JP, Couillard-Despres S, Cooper-Kuhn CM, Winkler J, Aigner L, Kuhn HG (2003). Transient expression of doublecortin during adult neurogenesis. J Comp Neurol 467: 1-10.

Bruel-Jungerman E, Laroche S, Rampon C (2005). New neurons in the dentate gyrus are involved in the expression of enhanced long-term memory following environmental enrichment. Eur J Neurosci 21: 513-521.

Cameron HA, Tanapat P, Gould E (1998). Adrenal steroids and Nmethyl-D-aspartate receptor activation regulate neurogenesis in the dentate gyrus of adult rats through a common pathway. Neuroscience 82: 349-354.

Coluccia AM, Gunby RH, Tartari CJ, Scapozza L, GambacortiPasserini C, Passoni L (2005). Anaplastic lymphoma kinase and its signalling molecules as novel targets in lymphoma therapy. Expert Opin Ther Targets 9: 515-532. 
Cryan JF, Mombereau C (2004). In search of a depressed mouse: utility of models for studying depression-related behavior in genetically modified mice. Mol Psychiatry 9: 326-357.

Duyster J, Bai RY, Morris SW (2001). Translocations involving anaplastic lymphoma kinase (ALK). Oncogene 20: 5623-5637.

Felling RJ, Levison SW (2003). Enhanced neurogenesis following stroke. J Neurosci Res 73: 277-283.

Ferrari K, Giuliani D (1997). Effects of (-)eticlopride and 7-OHDPAT $\mathrm{n}$ the tail suspension test in mice. J Psychopharmacology 11: $339-344$.

Furuta M, Shiraishi T, Okamoto H, Mineta T, Tabuchi K, Shiwa M (2004). Identification of pleiotrophin in conditioned medium secreted from neural stem cells by SELDI-TOF and SELDItandem mass spectrometry. Brain Res Dev Brain Res 152: 189-197.

Gould E, Tanapat P (1997). Lesion-induced proliferation of neuronal progenitors in the dentate gyrus of the adult rat. Neuroscience 80: 427-436.

Hida H, Jung C, Wu C, Kim H, Kodama Y, Masuda T et al (2003). Pleiotrophin exhibits a trophic effect on survival of doapminergic neurons in vitro. Eur J Neurosc 17: 2127-2134.

Hoglinger GU, Rizk P, Muriel MP, Duyckaerts C, Oertel WH, Caille I et al (2004). Dopamine depletion impairs precursor cell proliferation in Parkinson disease. Nat Neurosci 7: 726-735.

Holmberg J, Armulik A, Senti KA, Edoff K, Spalding K, Momma S et al (2005). Ephrin-A2 reverse signaling negatively regulates neural progenitor proliferation and neurogenesis. Genes Dev 19: 462-471.

Hutson PH, Bristow LJ, Thorn L, Tricklebank MD (1991). R-(+)HA-966, a glycine/NMDA receptor antagonist, selectively blocks the activation of the mesolimbic dopamine system by amphetamine. Br J Pharmacol 103: 2037-2044.

Iwahara T, Fujimoto J, Wen D, Cupples R, Bucay N, Arakawa T et al (1997). Molecular characterization of ALK, a receptor tyrosine kinase expressed specifically in the nervous system. Oncogene 14: 439-449.

Jung CG, Hida H, Nakahira K, Ikenaka K, Kim HJ, Nishino H (2004). Pleiotrophin mRNA is highly expressed in neural stem (progenitor) cells of mouse ventral mesencephalon and the product promotes production of dopaminergic neurons from embryonic stem cell-derived nestin-positive cells. FASEB J 18: 1237-1239.

Kempermann G, Jessberger S, Steiner B, Kronenberg G (2004b). Milestones of neuronal development in the adult hippocampus. Trends Neurosci 27: 447-452.

Kempermann G, Wiskott L, Gage FH (2004a). Functional significance of adult neurogenesis. Curr Opin Neurobiol 14: 186-191.

Kempermann G, Gage FH (1999). Experience-dependent regulation of adult hippocampal neurogenesis: effects of long-term stimulation and stimulus withdrawal. Hippocampus 9: 321-332.

Kempermann G (2002). Regulation of adult hippocampal neurogenesis - implications for novel theories of major depression. Bipolar Disord 4: 17-33.

Kinnunen T, Raulo E, Nolo R, Maccarana M, Lindahl U, Rauvala H (1996). Neurite outgrowth in brain neurons induced by heparinbinding growth-associated molecule (HB-GAM) depends on the specific interaction of HB-GAM with heparan sulfate at the cell surface. J Biol Chem 271: 2243-2248.

Kippin TE, Kapur S, van der Kooy D (2005). Dopamine specifically inhibits forebrain neural stem cell proliferation, suggesting a novel effect of antipsychotic drugs. J Neurosci 25: 5815-5823.

Kunugi H, Hashimoto R, Okada T, Hori H, Nakabayashi T, Baba A et al (2006). Possible association between nonsynonymous polymorphisms of the anaplastic lymphoma kinase (ALK) gene and schizophrenia in a Japanese population. J Neural Transmission 113: 1569-1573.

Leuner B, Mendolia-Loffredo S, Kozorovitskiy Y, Samburg D, Gould E, Shors TJ (2004). Learning enhances the survival of new neurons beyond the time when the hippocampus is required for memory. J Neurosci 24: 7477-7481.

Lie DC, Song H, Colamarino SA, Ming GL, Gage FH (2004). Neurogenesis in the adult brain: new strategies for central nervous system diseases. Annu Rev Pharmacol Toxicol 44: 399-421.

Louissaint A, Rao S, Leventhal C, Goldman SA (2002). Coordinated interaction of neurogenesis and angiogenesis in the adult songbird brain. Neuron 34: 945-960.

Maeda N, Ichihara-Tanaka K, Kimura T, Kadomatsu K, Muramatsu T, Noda M (1999). A receptor-like protein-tyrosine phosphatase PTPzeta/RPTPbeta binds a heparin-binding growth factor midkine. Involvement of arginine 78 of midkine in the high affinity binding to PTPzeta. J Biol Chem 274: 12474-12479.

Magavi SS, Leavitt BR, Macklis JD (2000). Induction of neurogenesis in the neocortex of adult mice. Nature 405: 951-955.

Mandillo S, Rinaldi A, Oliverio A, Mele A (2003). Repeated administration of phencyclidine, amphetamine and MK-801 selectively impairs spatial learning in mice: a possible model of psychotomimetic drug-induced cognitive deficits. Behav Pharmacol 14: 533-544.

McEwen BS, Olie JP (2005). Neurobiology of mood, anxiety, and emotions as revealed by studies of a unique antidepressant: tianeptine. Mol Psychiatry 10: 525-537.

Meng K, Rodriguez-Pena A, Dimitrov T, Chen W, Yamin M, Noda $\mathrm{M}$, et al (2000). Pleiotrophin signals increased tyrosine phosphorylation of beta beta-catenin through inactivation of the intrinsic catalytic activity of the receptor-type protein tyrosine phosphatase beta/zeta. Proc Natl Acad Sci USA 97: 2603-2608.

Mirescu C, Peters JD, Gould E (2004). Early life experience alters response of adult neurogenesis to stress. Nat Neurosci 7: 841-846.

Monje ML, Toda H, Palmer TD (2003). Inflammatory blockade restores adult hippocampal neurogenesis. Science 302: $1760-1765$.

Mori A, Ohashi S, Nakai M, Moriizumi T, Mitsumoto Y (2005). Neural mechanisms underlying motor dysfunction as detected by the tail suspension test in MPTP-treated C57BL/6 mice. Neurosci Res 51: 265-274.

Morris SW, Naeve C, Mathew P, James PL, Kirstein MN, Cui X et al (1997). ALK, the chromosome 2 gene locus altered by the $t(2 ; 5)$ in non-Hodgkin's lymphoma, encodes a novel neural receptor tyrosine kinase that is highly related to leukocyte tyrosine kinase (LTK). Oncogene 14: 2175-2188.

Nakamura E, Kadomatsu K, Yuasa S, Muramatsu H, Mamiya T, Nabeshima T et al (1998). Disruption of the midkine gene (Mdk) resulted in altered expression of a calcium binding protein in the hippocampus of infant mice and their abnormal behaviour. Genes Cells 3: 811-822.

Nakatomi H, Kuriu T, Okabe S, Yamamoto S, Hatano O, Kawahara $\mathrm{N}$ et al (2002). Regeneration of hippocampal pyramidal neurons after ischemic brain injury by recruitment of endogenous neural progenitors. Cell 110: 429-441.

Ohtani N, Goto T, Waeber C, Bhide PG (2003). Dopamine modulates cell cycle in the lateral ganglionic eminence. J Neurosci 23: $2840-2850$.

Osajima-Hakomori Y, Miyake I, Ohira M, Nakagawara A, Nakagawa A, Sakai R (2005). Biological role of anaplastic lymphoma kinase in neuroblastoma. Am J Pathol 167: 213-222.

Pavlov I, Voikar V, Kaksonen M, Lauri SE, Hienola A, Taira T et al (2002). Role of heparin-binding growth-associated molecule (HB-GAM) in hippocampal LTP and spatial learning revealed by studies on overexpressing and knockout mice. Mol Cell Neurosci 20: 330-342.

Rao MS, Shetty AK (2004). Efficacy of doublecortin as a marker to analyse the absolute number and dendritic growth of newly generated neurons in the adult dentate gyrus. Eur J Neurosci 19: 234-246. 
Sakaguchi N, Muramatsu H, Ichihara-Tanaka K, Maeda N, Noda M, Yamamoto $\mathrm{T}$ et al (2003). Receptor-type protein tyrosine phosphatase zeta as a component of the signaling receptor complex for midkine-dependent survival of embryonic neurons. Neurosci Res 45: 219-224.

Santarelli L, Saxe M, Gross C, Surget A, Battaglia F, Dulawa S et al (2003). Requirement of hippocampal neurogenesis for the behavioral effects of antidepressants. Science 301: 805-809.

Shi SR, Key ME, Kalra KL (1991). Antigen retrieval in formalinfixed, paraffin-embedded tissues: an enhancement method for immunohistochemical staining based on microwave oven heating of tissue sections. J Histochem Cytochem 39: 741-748.

Shors TJ, Miesegaes G, Beylin A, Zhao M, Rydel T, Gould E (2001). Neurogenesis in the adult is involved in the formation of trace memories. Nature 410: 372-376.

Siever LJ, Davis KL (2004). The pathophysiology of schizophrenia disorders: perspectives from the spectrum. Am J Psychiatry 161: 398-413.

Snyder JS, Hong NS, McDonald RJ, Wojtowicz JM (2005). A role for adult neurogenesis in spatial long-term memory. Neuroscience 130: 843-852.
Vega CJ, Peterson DA (2005). Stem cell proliferative history in tissue revealed by temporal halogenated thymidine analog discrimination. Nat Methods 2: 167-169.

Vernersson E, Khoo NK, Henriksson ML, Roos G, Palmer RH, Hallberg B (2006). Characterization of the expression of the ALK receptor tyrosine kinase in mice. Gene Expr Patterns 6: 448-461.

Winkler S, Stahl RC, Carey DJ, Bansal R (2002). Syndecan-3 and perlecan are differentially expressed by progenitors and mature oligodendrocytes and accumulate in the extracellular matrix. J Neurosci Res 69: 477-487.

Yang CR, Chen L (2005). Targeting prefrontal cortical dopamine D1 and $N$-methyl-D-aspartate receptor interactions in schizophrenia treatment. Neuroscientist 11: 452-468.

Zhang R, Zhang Z, Zhang C, Zhang L, Robin A, Wang Y et al (2004). Stroke transiently increases subventricular zone cell division from asymmetric to symmetric and increases neuronal differentiation in the adult rats. J Neurosci 24: 5810-5815.

Zhou H, Muramatsu T, Halfter W, Tsim KW, Peng HB (1997). A role of midkine in the development of the neuromuscular junction. Mol Cell Neurosci 10: 56-70. 